\title{
The Bending-Gradient Theory for Thick Plates: Existence and Uniqueness Results
}

\author{
Nadine Bejjani • Karam Sab • Joanna Bodgi • \\ Arthur Lebée
}

Received: date / Accepted: date

\begin{abstract}
This paper is devoted to the mathematical justification of the Bending-Gradient theory which is considered as the extension of the Reissner-Mindlin theory (or the First Order Shear Deformation Theory) to heterogeneous plates. In order to rigorously assess the well-posedness of the Bending-Gradient problems, we first assume that the compliance tensor related to the generalized shear force is positive definite. We define the functional spaces to which the variables of the theory belong, then state and prove the existence and uniqueness theorems of solutions of the Bending-Gradient problems for clamped and free plates, as well as for simply supported plates. The obtained results are afterward extended to the general case, i.e., when the compliance tensor related to generalized shear forces is not definite.
\end{abstract}

Keywords Plate theory $\cdot$ Bending-Gradient theory $\cdot$ Heterogeneous plates $\cdot$ Mathematical Justification - Boundary conditions · Existence and uniqueness of solution · Variational methods

Mathematics Subject Classification (2010) 74G65 · 74K20

\section{Introduction}

Plates are three-dimensional structures with a small dimension compared to the other two dimensions. Numerous approaches were suggested in order to replace the three-dimensional problem by a two-dimensional problem while guaranteeing accuracy of the three-dimensional fields' information. The passage from the $3 \mathrm{D}$ problem to a $2 \mathrm{D}$ plate theory is known as dimensional reduction. It also makes the theory easier to understand and to deal with. Every two-dimensional plate theory is indeed judged based on how well its solution approximates

N. Bejjani · K. Sab · A. Lebée

Laboratoire Navier, UMR 8205, École des Ponts ParisTech, IFSTTAR, CNRS, Université Paris-Est

6-8 av. Blaise Pascal, Cité Descartes, 77420 Champs sur Marne, France

E-mail: \{arthur.lebee ; karam.sab ; nadine.bejjani\}@enpc.fr

N. Bejjani · J. Bodgi

Unité de recherche « Mathématiques et modélisation », Faculté des sciences, Université Saint-Joseph,

B.P. 11-514 Riad El Solh Beyrouth 1107 2050, Lebanon.

E-mail: joanna.bodgi@usj.edu.lb 
the corresponding three-dimensional problem. The most common dimensionally reduced theories of thin plates are the Kirchhoff-Love and the Reissner-Mindlin theories. The mathematical justification of these plate theories is hence interesting and has a long history. It has been a challenging subject to engineers and mathematicians throughout the past centuries. Two distinct ways to justify two-dimensional plate theories are either by using asymptotic expansions or by using variational techniques.

The Kirchhoff-Love theory, known also as the classical theory of plates, is an extension of Euler-Bernoulli beam theory. It dates back to 1888 and is based on the assumption that straight lines normal to the mid-plane of the plate remain normal after deformation. Morgenstern was the first to prove in 1959 that the Kirchhoff theory is correct for thin plates, i.e., when the thickness approaches zero (see [21]). His analysis was performed using the two-energies principle of Prager and Synge, known also as the hypercircle theorem ([27]). Next, Ciarlet and Destuynder established rigorous error estimates between the solution of the 3D elastic problem and its limit (see [9,7]). Later on, another mathematical justification of the Kirchhoff-Love theory was established by Ciarlet using convergence theorems as the thickness of the plate approaches zero (see [8] for more details).

Due to its normality assumption, the classical plate theory neglects deformations caused by transverse shear. This leads to considerable errors when applied to moderately thick plates. Reissner [29], Hencky [11] and Bollé [4] independently developed plate bending formulations trying to eliminate the above-mentioned deficiency of Kirchhoff-Love plate theory and gathered here under the usual denomination Reissner-Mindlin theories. To this aim, they released Kirchhoff's normality constraint, i.e., straight lines normal to the midplane of the plate undergo an independent rotation after deformation. This allowed them to take into account shear deformations through the thickness of the plate. The Reissner-Mindlin theory is often called the first-order shear deformation theory (FOSDT). This theory was proved to have a wider range of applicability than the Kirchhoff-Love theory particularly for clamped plates of small to moderate thickness when transverse shear plays a significant role (see [31] and [12]). According to [31], the Reissner-Mindlin theory is also preferred because it better represents boundary conditions since it can distinguish between hard and soft simple support conditions.

There is a vast literature regarding Reissner-Mindlin theory. Indeed, The Kirchhoff-Love theory is the limit model of the Reissner-Mindlin theory when the thickness of the plate goes to 0 . This limit behavior enforces a jump of regularity of the solution and the emergence of boundary layers which required specific numerical treatments (see for instance [2, 1, 3, 5]. Furthermore, rigorous derivations of the Reissner-Mindlin model as well as proof of higherorder convergence of the Reissner-Mindlin remain under discussion (see [5, 26, 22] among others).

Reissner-Mindlin's theory is widely used in applied mechanics since it works well for homogeneous plates. However, applying it directly to laminated plates leads to discontinuous transverse shear distributions in addition to incorrect estimation of the deflection compared to exact solutions. In recent decades, many studies have been conducted in order to try to capture correctly the effects of transverse shear deformations (see [34, 28, 23, 10, 6]). Recently, motivated and inspired by the ideas and techniques presented in [30], A. Lebée and $\mathrm{K}$. Sab derived a new plate theory called the Bending-Gradient theory for thick heterogeneous plates ([13]). This theory replaces the classical Reissner-Mindlin out-of-plane shear force by a generalized shear force related to the first gradient of the bending moment. The BendingGradient theory is hence considered as an extension to laminated plates of the ReissnerMindlin plate theory. A. Lebée and K. Sab also demonstrated that the Bending-Gradient theory cannot be reduced to a Reissner-Mindlin theory unless the plate under consideration 
is homogeneous (see [13]). The new theory was compared to the Reissner-Mindlin theory and to full 3D ([24, 25]) exact solutions in [14]. They came up with the conclusion that their new theory gives good prediction of deflection, shear stress distributions and in-plane displacement distributions in any material configuration. They also extended their new theory, which was originally designed for laminated plates, to in-plane periodic plates and they applied it to sandwich panels (see [16] and [15]), as well as space frames ([17]). Finally, the Bending-Gradient theory was justified through asymptotic expansions [18].

We concentrate in this paper on the mathematical justification of this theory by means of variational methods. The main emphasis is put on clamped and free plates. We are principally concerned with identifying mathematical spaces in which the corresponding variational problems are well-defined, in order to formulate existence and uniqueness theorems for the solutions of these problems. For full details on the Bending-Gradient theory, we refer to [32].

The paper proceeds as follows. Section 2 provides a brief review of tensor calculus and necessary notions that will be needed in this work. Furthermore, it recalls some well-known definitions in addition to theorems and results which are essential for the problems that we want to consider. In Section 3, we set briefly the 3D elastic problem for a clamped plate then we summarize synthetically the Bending-Gradient problem. We propose next in Section 4 a proper mathematical framework for the Bending-Gradient theory. Section 4.1 is devoted to the stress formulation of the new plate theory then to the proof of its well-posedness (Theorem 11. In Section 4.3, we present a displacement formulation which is based on the minimum potential energy principle. In Section 5, we study the problem with free boundary conditions and prove that it admits a unique solution (Theorem 47). In Section 6 we discuss the problem when the plate is simply supported. It should be underlined that throughout Sections 46 we treat the particular case where the compliance tensor related to the gradient of the bending moment is positive definite. In Section 7, we extend the obtained results to the general case and provide corresponding stress and displacement formulations. Section 8 is intended to provide a regularization of the Bending-Gradient problem and to study the convergence of the regularized solution to the exact solution of the problem in the general case. Finally, we conclude in Section 9 with some final remarks.

\section{Notations and preliminaries}

In this section, we introduce our notations and we recall some existing results which are crucial for our analysis.

\subsection{Tensors}

Throughout this paper, we shall assume that all vector spaces are over $\mathbb{R}$. First, second, third, fourth and sixth rank tensors are denoted by $\underline{\boldsymbol{X}}, \underset{\sim}{\boldsymbol{X}}, \underset{\sim}{\boldsymbol{X}}, \underset{\sim}{\boldsymbol{X}}, \underset{\approx}{\boldsymbol{X}}$, respectively. They comply with specific symmetries detailed in the following. Note that these notations are used for both $2 D$ and $3 D$ tensors. For tensor components, we use Greek indices to represent $2 D$ tensors $\left(\alpha, \beta, \gamma_{. .}=1,2\right)$ and Latin indices to represent $3 D$ tensors $(i, j, k . .=1,2,3)$. For example, $\left(X_{i j}\right)$ denotes the $3 D$ tensor $\underset{\sim}{\boldsymbol{X}}$ whereas $\left(X_{\alpha \beta}\right)$ designates the $2 D$ tensor $\underset{\sim}{\boldsymbol{X}}$. For simplicity purposes, we adopt the Einstein summation convention on repeated indices when manipulating expressions involving tensors.

The transpose operation ${ }^{T} \bullet$ is applied to any order tensors as follows: $\left({ }^{T} X\right)_{\alpha \beta \ldots \psi \omega}=$ $X_{\omega \psi \ldots \beta \alpha}$. Four symbols are defined: (·), (:), (:) and (!) for contraction on, respectively, one, 
two, three and four indices. By convention, the closest indices are successively summed together in contraction products. Thus, $\underset{\boldsymbol{X}}{\boldsymbol{X}} \underline{\boldsymbol{Y}}=\left(X_{\alpha \beta \gamma \delta \lambda \mu} Y_{\mu \lambda \delta}\right)$ and $\underset{\boldsymbol{X}}{\boldsymbol{Y}} \underline{\boldsymbol{Y}}=\left(X_{\alpha \beta \gamma} Y_{\gamma}\right)$ is different from $\underline{\boldsymbol{Y}} \cdot \underline{\boldsymbol{X}}=\left(Y_{\alpha} X_{\alpha \beta \gamma}\right)$.

The identity for $2 D$ vectors is $\underset{\sim}{\delta}=\left(\delta_{\alpha \beta}\right)$ where $\delta_{\alpha \beta}$ is Kronecker symbol $\left(\delta_{\alpha \beta}=1\right.$ if $\alpha=\beta, \delta_{\alpha \beta}=0$ otherwise). The identity for $2 D$ symmetric second order tensors is $\underset{\sim}{\boldsymbol{i}}$ where $i_{\alpha \beta \gamma \delta}=\frac{1}{2}\left(\delta_{\alpha \gamma} \delta_{\beta \delta}+\delta_{\alpha \delta} \delta_{\beta \gamma}\right)$. The reader might easily check that $\underset{\sim}{i}: \underset{\sim}{i}=\underset{\sim}{i} \underset{\sim}{i}: \underset{\sim}{i}=3 / 2 \underset{\sim}{\delta}$ and $\underset{\sim}{i} \underset{\sim}{i}=3$.

The gradient of a scalar field $X$ writes $\underline{\nabla} X=\left(X_{, \beta}\right)$ while the gradient of a vector or a higher-order tensor fields writes $\underset{\sim}{\boldsymbol{X}} \otimes \underline{\boldsymbol{\nabla}}=\left(X_{\alpha \beta, \gamma}\right)$, for instance, where $\otimes$ is the dyadic product. The divergence of a vector field or a second order tensor field is noted $\underline{\boldsymbol{X}} \cdot \underline{\boldsymbol{\nabla}}=\left(X_{\alpha, \alpha}\right)$ and $\underset{\sim}{\boldsymbol{X}} \cdot \underline{\boldsymbol{\nabla}}=\left(X_{\alpha \beta, \beta}\right)$, respectively.

Let $\underline{\mathbb{R}}, \underset{\mathbb{R}}{\mathbb{R}}$ and $\mathbb{R}$ be the spaces of $2 D$ first, second and third rank tensors which comply with the following symmetries:

$$
\begin{gathered}
\underset{\sim}{\mathbb{R}}=\left\{\underline{X}=\left(X_{\alpha \beta}\right) \in \mathbb{R}^{4} \mid X_{\alpha \beta}=X_{\beta \alpha}\right\}, \\
\stackrel{\mathbb{R}}{\mathbb{R}}=\left\{\underline{X}=\left(X_{\alpha \beta \gamma}\right) \in \mathbb{R}^{8} \mid X_{\alpha \beta \gamma}=X_{\beta \alpha \gamma}\right\} .
\end{gathered}
$$

The spaces $\underset{\sim}{\mathbb{R}}$ and $\underset{\mathbb{R}}{\mathbb{R}}$ are respectively endowed with the following scalar products:

$$
{ }^{T} \underset{\sim}{X}: \underset{\sim}{\boldsymbol{X}^{\prime}}=X_{\alpha \beta} X_{\alpha \beta}^{\prime},
$$

and

$$
{ }^{T} \underset{X}{X}: \underline{X}^{\prime}=X_{\alpha \beta \gamma} X_{\alpha \beta \gamma}^{\prime} .
$$

Any $2 D$ third-rank tensor $\underset{X}{\boldsymbol{X}}$ in $\underset{\mathbb{R}}{\mathbb{R}}$ can be orthogonally decomposed into a spherical part $\underline{X}^{\mathrm{s}}$ and a deviatori ${ }^{1}$ part $\underline{X}^{\mathrm{d}}$ as:

$$
\underline{\boldsymbol{X}}=\underline{\boldsymbol{X}}^{\mathrm{s}}+\underline{X}^{\mathrm{d}}
$$

where

$$
X_{\alpha \beta \gamma}^{\mathrm{s}}=\frac{1}{3}\left(X_{\alpha \nu \lambda} \delta_{v \lambda} \delta_{\beta \gamma}+X_{\beta \nu \lambda} \delta_{v \lambda} \delta_{\alpha \gamma}\right) .
$$

One can easily check the following properties:

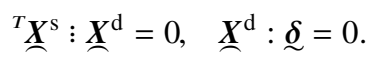

Note that any tensor of the form :

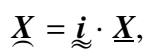

where $\underline{X} \in \underline{\mathbb{R}}$, is a purely spherical tensor. Conversely, for any given $\underline{X} \in \underline{\mathbb{R}}$, there exists a unique vector $\underline{\boldsymbol{X}}^{\mathrm{s}}$ extracted from its spherical part such that $(3)$ is satisfied for $\underline{\boldsymbol{X}}^{\mathrm{s}}$. This vector is given by:

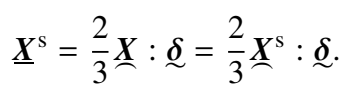

1 Whereas the spherical and deviatoric part of a second rank tensor is conventional, this denomination is understood here for the two last indices of a third rank tensor. 


\subsection{Functional spaces}

Let $\omega$ be a bounded, connected, open subset of $\mathbb{R}^{2}$ with a Lipschitz-continuous boundary. We denote by $C^{\infty}(\omega)$ the space of infinitely differentiable functions on $\omega$ and by $D(\omega)$ the subspace of functions with compact support in $\omega$. We further refer to $D^{\prime}(\omega)$ as the space of distributions on $\omega$. The fundamental Sobolev space $L^{2}(\omega)$ is the space of square integrable functions on $\omega$. For each integer $m \geq 1, H^{m}(\omega)$ and $H_{0}^{1}(\omega)$ denote the usual Sobolev spaces. To avoid ambiguity, we will use the tensorial notations as mentioned in section 2.1 Namely, $\underline{\boldsymbol{L}}^{2}(\omega), \underline{\boldsymbol{L}}^{2}(\omega)$ and $\underline{\boldsymbol{L}}^{2}(\omega)$ denote, respectively, the space of vector fields, symmetric second rank fields and symmetric third rank fields having their components in $L^{2}(\omega)$. The associated norms are defined as:

$$
\|\underline{\boldsymbol{a}}\|_{\underline{\boldsymbol{L}}^{2}(\omega)}^{2}=\int_{\omega} \underline{\boldsymbol{a}} \cdot \underline{\boldsymbol{a}}, \quad\|\underline{\boldsymbol{a}}\|_{\underline{\boldsymbol{L}}^{2}(\omega)}^{2}=\int_{\omega} \underset{\boldsymbol{a}}{2}: \underline{\boldsymbol{a}}, \quad\|\underline{\boldsymbol{a}}\|_{\underline{\boldsymbol{L}}^{2}(\omega)}^{2}=\int_{\omega} \underline{\boldsymbol{a}}: \underline{\boldsymbol{a}} .
$$

Here, we have used the fact that tensor fields $\underset{\boldsymbol{a}}{\mathrm{o}}$ of $\underset{\sim}{\boldsymbol{L}^{2}}(\omega)$ are symmetric, i.e., ${ }^{T} \boldsymbol{a}=\underset{\sim}{\boldsymbol{a}}$. The well-posedness of the Bending-Gradient problems relies on some intermediate theorems and results which we state here as Lemmas. First, Lemma 1 to Lemma 4 have been already established for three-dimensional tensor fields in [33] and their straightforward extension to the case of two-dimensional tensors will not be detailed. Then, Lemma 5 is an original contribution which will be proved below.

Lemma 1 The set defined by:

$$
\underline{H}(\operatorname{div}, \omega)=\left\{\underline{\boldsymbol{\Phi}} \in \underline{\boldsymbol{L}}^{2}(\omega) ; \quad \underline{\boldsymbol{\Phi}} \cdot \underline{\boldsymbol{\nabla}} \in \underline{\boldsymbol{L}}^{2}(\omega)\right\},
$$

equipped with the scalar product :

$$
\left\langle\underline{\Phi}_{1}, \Phi_{2}\right\rangle_{\underline{H}(d i v, \omega)}=\int_{\omega}{ }^{T} \Phi_{1}: \Phi_{2}+\left(\Phi_{1} \cdot \underline{\nabla}\right):\left(\Phi_{2} \cdot \underline{\nabla}\right),
$$

and its associated norm $\|\bullet\|_{\underline{\boldsymbol{H}}(\text { div, } \omega)}$ is a Hilbert space.

We now give an appropriate sense to the Dirichlet boundary condition related to $\boldsymbol{H}(\operatorname{div}, \omega)$ : $\underline{\Phi} \cdot \underline{\boldsymbol{n}}=0$. It is well-known that we can define the trace of $\underline{H}^{1}(\omega)$ tensors on $\partial \omega$. However, the trace of a tensor belonging to $\underline{\boldsymbol{L}}^{2}(\omega)$ is not defined. Since $\boldsymbol{\Phi} \in \boldsymbol{H}(\operatorname{div}, \omega)$, it is less regular than a $\underline{H}^{1}(\omega)$ tensor. Nevertheless, we can set its normal trace $\underline{\Phi} \cdot \underline{\boldsymbol{n}}$ to zero in the

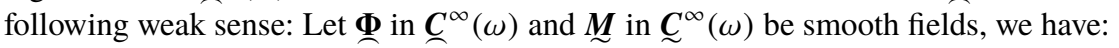

$$
\int_{\omega} \underset{\sim}{\boldsymbol{M}}:(\underline{\boldsymbol{\Phi}} \cdot \underline{\boldsymbol{\nabla}})+{ }^{T}(\underset{\sim}{\boldsymbol{M}} \otimes \underline{\boldsymbol{\nabla}}): \underline{\boldsymbol{\Phi}}=\int_{\partial \omega} \underset{\sim}{\boldsymbol{M}}:(\underline{\boldsymbol{\Phi}} \cdot \underline{\boldsymbol{n}}) .
$$

Since (7) holds true for any given $\underset{\sim}{\boldsymbol{M}}$ on $\partial \omega$, imposing $\underline{\boldsymbol{\Phi}} \cdot \underline{\boldsymbol{n}}=0$ is thus equivalent to imposing:

$$
\int_{\omega} \underset{\sim}{\boldsymbol{M}}:(\underline{\boldsymbol{\Phi}} \cdot \underline{\boldsymbol{\nabla}})+{ }^{T}(\underline{\boldsymbol{M}} \otimes \underline{\boldsymbol{\nabla}}): \underline{\boldsymbol{\Phi}}=0,
$$

for all $\underset{\sim}{\boldsymbol{M}} \in \boldsymbol{C}^{\infty}(\omega)$. The following definition is hence very natural.

Lemma 2 The set defined by:

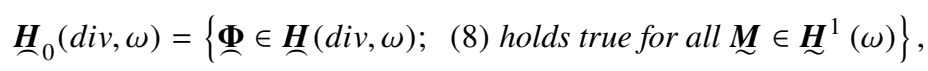

is a closed subspace of $\underline{\boldsymbol{H}}($ div, $\omega)$ equipped with its norm $\|\bullet\|_{\boldsymbol{H}(\operatorname{div}, \omega)}$. Hence, $\underline{\boldsymbol{H}}_{0}(\operatorname{div}, \omega)$ endowed with the scalar product $\langle\bullet, \bullet\rangle_{\boldsymbol{H}(\text { div, } \omega)}$ is a Hilbert space. 
Lemma 3 Let $\left(\Phi_{k}\right)_{k \in \mathbb{N}^{*}}$ be a sequence of third-rank tensors defined over $\underline{H}_{0}($ div, $\omega)$. We denote by $\boldsymbol{\Phi}_{k}^{\mathrm{d}}$ the deviatoric part of $\boldsymbol{\Phi}_{k}$. If $\boldsymbol{\Phi}_{k}$ satisfies :

$$
\left\|\underline{\Phi}_{k} \cdot \underline{\nabla}\right\|_{{\underline{\boldsymbol{L}^{2}(\omega)}}}+\left\|\underline{\Phi}_{k}^{\mathrm{d}}\right\|_{{\underline{\boldsymbol{L}^{2}(\omega)}}} \leq \frac{C}{k}
$$

where $C$ is a strictly positive constant, then $\Phi_{k}$ strongly converges to zero in $\boldsymbol{H}($ div, $\omega)$.

Lemma 4 There exists a strictly positive constant $C$ such that, for all $\boldsymbol{\Phi} \in \boldsymbol{H}(\operatorname{div}, \omega)$, there exists a two-dimensional rigid motion vector field $\underline{\boldsymbol{r}}$ of the form:

$$
\underline{\boldsymbol{r}}\left(x_{1}, x_{2}\right)=\left(u-w x_{2}, v+w x_{1}\right),
$$

such that:

$$
\left\|\underline{\Phi}^{\mathrm{s}}-\underline{\boldsymbol{r}}\right\|_{\underline{\boldsymbol{L}}^{2}(\omega)} \leq C\left(\|\underline{\boldsymbol{\Phi}} \cdot \underline{\boldsymbol{\nabla}}\|_{\underline{\boldsymbol{L}}^{2}(\omega)}^{2}+\left\|\underline{\boldsymbol{\Phi}}^{\mathrm{d}}\right\|_{\underline{\boldsymbol{L}}^{2}(\omega)}^{2}\right)^{1 / 2} .
$$

Here, $(u, v)$ and $w$ are respectively the in-plane rotation vector and the global out-of-plane twist of the rigid motion field $\underline{\boldsymbol{r}}, \Phi^{\mathrm{d}}$ denotes the deviatoric part of $\boldsymbol{\Phi}$ and $\boldsymbol{\Phi}^{\mathrm{s}}$ is the vector field associated to the spherical part of $\Phi$ through (4).

Lemma 5 Assume that for each integer $k$, there exists a vector field $\underline{\boldsymbol{r}}_{k}$ of the form $[10]$ :

$$
\underline{\boldsymbol{r}}_{k}\left(x_{1}, x_{2}\right)=\left(u_{k}-w_{k} x_{2}, v_{k}+w_{k} x_{1}\right),
$$

and a scalar field $W_{k}$ in $H^{1}(\omega)$ such that:

$$
\left\|\underline{\boldsymbol{r}}_{k}+\underline{\boldsymbol{\nabla}} W_{k}\right\|_{\underline{\boldsymbol{L}}^{2}(\omega)} \longrightarrow 0
$$

as $k$ goes to infinity. Then, $w_{k} \longrightarrow 0$ and there exists a sequence of real numbers denoted by $z_{k}$ such that:

$$
\left\|W_{k}+u_{k} x_{1}+v_{k} x_{2}+z_{k}\right\|_{H^{1}(\omega)} \longrightarrow 0 .
$$

Moreover, if $W_{k}$ is in $H_{0}^{1}(\omega)$ for all $k$, then $\left(u_{k}, v_{k}\right) \longrightarrow(0,0)$ and:

$$
\left\|W_{k}\right\|_{H_{0}^{1}(\omega)} \longrightarrow 0 .
$$

Proof Let us decompose $\underline{\boldsymbol{r}}_{k}$ into two terms $\underline{\boldsymbol{r}}_{k}=\underline{\boldsymbol{r}}_{k}^{(u, v)}+\underline{\boldsymbol{r}}_{k}^{w}$ with $\underline{\boldsymbol{r}}_{k}^{(u, v)}=\left(u_{k}, v_{k}\right)$ and $\underline{\boldsymbol{r}}_{k}^{w}=\left(-w_{k} x_{2}, w_{k} x_{1}\right)$. Then, the limit (12) can be written as:

$$
\left\|\underline{\boldsymbol{r}}_{k}^{w}+\underline{\boldsymbol{\nabla}}\left(W_{k}+u_{k} x_{1}+v_{k} x_{2}\right)\right\|_{\underline{\boldsymbol{L}}^{2}(\omega)} \longrightarrow 0 .
$$

Assume first that the origin point $(0,0)$ is inside the domain $\omega$. Since $\omega$ is open, there exists a disc $D_{\rho}$ in $\omega$ of radius $\rho$ centered at $(0,0)$. We have:

$$
\begin{aligned}
& \left\|\underline{\boldsymbol{r}}_{k}^{w}+\underline{\boldsymbol{\nabla}}\left(W_{k}+u_{k} x_{1}+v_{k} x_{2}\right)\right\|_{\underline{\boldsymbol{L}}^{2}(\omega)}^{2} \geq\left\|\underline{\boldsymbol{r}}_{k}^{w}+\underline{\boldsymbol{\nabla}}\left(W_{k}+u_{k} x_{1}+v_{k} x_{2}\right)\right\|_{\underline{\boldsymbol{L}}^{2}\left(D_{\rho}\right)}^{2}= \\
& \left\|\underline{\boldsymbol{r}}_{k}^{w}\right\|_{\underline{\boldsymbol{L}}^{2}\left(D_{\rho}\right)}^{2}+\left\|\underline{\boldsymbol{\nabla}}\left(W_{k}+u_{k} x_{1}+v_{k} x_{2}\right)\right\|_{\underline{\boldsymbol{L}}^{2}\left(D_{\rho}\right)}^{2}+\int_{D_{\rho}} 2 \underline{\boldsymbol{r}}_{k}^{w} \cdot \underline{\boldsymbol{\nabla}}\left(W_{k}+u_{k} x_{1}+v_{k} x_{2}\right) .
\end{aligned}
$$

Integrating by parts the last term in the above right-hand side yields:

$$
\begin{aligned}
\int_{D_{\rho}} 2 \underline{\boldsymbol{r}}_{k}^{w} \cdot \underline{\boldsymbol{\nabla}}\left(W_{k}+u_{k} x_{1}+v_{k} x_{2}\right) & =-\int_{D_{\rho}} 2\left(\underline{\boldsymbol{r}}_{k}^{w} \cdot \underline{\boldsymbol{\nabla}}\right)\left(W_{k}+u_{k} x_{1}+v_{k} x_{2}\right) \\
& +\int_{\partial D_{\rho}} 2\left(\underline{\boldsymbol{r}}_{k}^{w} \cdot \underline{\boldsymbol{n}}\right)\left(W_{k}+u_{k} x_{1}+v_{k} x_{2}\right)=0
\end{aligned}
$$


because $\underline{\boldsymbol{r}}_{k}^{w} \cdot \underline{\boldsymbol{\nabla}}=0$ in the bulk and $\underline{\boldsymbol{r}}_{k}^{w} \cdot \underline{\boldsymbol{n}}=0$ at the boundary, where $\underline{\boldsymbol{n}}$ denotes the outer normal to $D_{\rho}$. Moreover, by a simple calculus, we obtain that $\left\|\underline{\underline{r}}_{k}^{w}\right\|_{\underline{\underline{L}}^{2}\left(D_{\rho}\right)}^{2}=\frac{\pi}{2} \rho^{4} w_{k}^{2}$. Consequently, by the limit $(15)$, we prove that $w_{k} \longrightarrow 0$ when the origin point is inside $\omega$.

Now, if the origin point $(0,0)$ is not inside the domain $\omega$, we can make the change of variables :

$$
\left(x_{1}, x_{2}\right)=\left(x_{1}^{\prime}, x_{2}^{\prime}\right)+\left(c_{1}, c_{2}\right)
$$

where $\left(c_{1}, c_{2}\right)$ is a point inside the domain $\omega$ and $\left(x_{1}^{\prime}, x_{2}^{\prime}\right)$ belongs to the translated domain $\omega^{\prime}$. Then, we define on the domain $\omega^{\prime}$ the fields:

$$
\underline{\boldsymbol{r}}_{k}^{\prime}\left(x_{1}^{\prime}, x_{2}^{\prime}\right)=\underline{\boldsymbol{r}}_{k}\left(x_{1}, x_{2}\right)=\left(u_{k}^{\prime}-w_{k}^{\prime} x_{2}^{\prime}, v_{k}^{\prime}+w_{k}^{\prime} x_{1}^{\prime}\right)
$$

and

$$
W_{k}^{\prime}\left(x_{1}^{\prime}, x_{2}^{\prime}\right)=W_{k}\left(x_{1}, x_{2}\right)
$$

with

$$
\left(u_{k}^{\prime}, v_{k}^{\prime}, w_{k}^{\prime}\right)=\left(u_{k}-w_{k} c_{2}, v_{k}+w_{k} c_{1}, w_{k}\right) .
$$

Then, by construction, the origin point $(0,0)$ is inside the domain $\omega^{\prime}$. Applying the above result to the fields $\underline{\boldsymbol{r}}_{k}^{\prime}$ and $W_{k}^{\prime}$, we prove that $w_{k} \longrightarrow 0$ in the general case and, consequently, the limit 15 becomes:

$$
\left\|\underline{\nabla} W_{k}+\underline{\boldsymbol{r}}_{k}^{(u, v)}\right\|_{\underline{\boldsymbol{L}}^{2}(\omega)}=\left\|\underline{\boldsymbol{\nabla}}\left(W_{k}+u_{k} x_{1}+v_{k} x_{2}\right)\right\|_{\underline{\mathbf{L}}^{2}(\omega)} \longrightarrow 0 .
$$

Using the well-known Poincaré inequality, we prove that there exists a sequence of real numbers denoted by $z_{k}$ such that 13 .

In the special case where $W_{k}$ is in $H_{0}^{1}(\omega)$ for all $k$, then using standard integration by parts gives:

$$
\left\|\underline{\nabla} W_{k}+\underline{\boldsymbol{r}}_{k}^{(u, v)}\right\|_{\underline{\boldsymbol{L}}^{2}(\omega)}^{2}=\left\|\underline{\boldsymbol{\nabla}} W_{k}\right\|_{\underline{\boldsymbol{L}}^{2}(\omega)}^{2}+\left\|\underline{\boldsymbol{r}}_{k}^{(u, v)}\right\|_{\underline{\boldsymbol{L}}^{2}(\omega)}^{2} .
$$

Hence, the limit 18$)$ implies $\left(u_{k}, v_{k}\right) \longrightarrow(0,0)$ and 14 by the Poincaré inequality.

\section{The Bending-Gradient problem}

In this section, we first set the $3 D$ laminated plate configuration. Then, we provide an overview of its reduction to a $2 D$ problem according to the Bending-Gradient theory. The definitions of the generalized stresses and strains of this theory are recalled, as well as the compatibility conditions, the equilibrium equations and the constitutive equations. We finally present the variational formulations of this linear problem without specifying the functional spaces to which must belong the unknown kinematic and static fields.

\subsection{The 3D configuration}

The physical space is endowed with an orthonormal reference $\left(O, \underline{\boldsymbol{e}}_{1}, \underline{\boldsymbol{e}}_{2}, \underline{\boldsymbol{e}}_{3}\right)$ where $O$ is the origin and $\underline{\boldsymbol{e}}_{i}$ is the base vector in direction $i \in\{1,2,3\}$. We consider a linear elastic plate occupying the $3 D$ domain $\Omega=\omega \times]-\frac{t}{2}, \frac{t}{2}\left[\right.$, where $\omega \subset \mathbb{R}^{2}$ is the middle surface of the 
plate and $t$ its thickness. The boundary of the domain, denoted by $\partial \Omega$, is decomposed intro three parts (Figure 1):

$$
\begin{gathered}
\partial \Omega=\partial \Omega_{\mathrm{lat}} \cup \partial \Omega_{3}^{+} \cup \partial \Omega_{3}^{-}, \\
\text {with } \left.\partial \Omega_{\text {lat }}=\partial \omega \times\right]-\frac{t}{2}, \frac{t}{2}\left[, \quad \text { and } \quad \partial \Omega_{3}^{ \pm}=\omega \times\left\{ \pm \frac{t}{2}\right\}\right. \text {, }
\end{gathered}
$$

where $\partial \omega$ is the boundary of $\omega$.

Focusing only on out-of-plane loadings, the plate is subjected to forces per unit surface on $\partial \Omega_{3}^{ \pm}$of the form:

$$
\underline{\boldsymbol{T}}^{ \pm}\left(x_{1}, x_{2}\right)=\left(0,0, \frac{1}{2} p\left(x_{1}, x_{2}\right)\right),
$$

where $p$ is a given function on $\omega$.

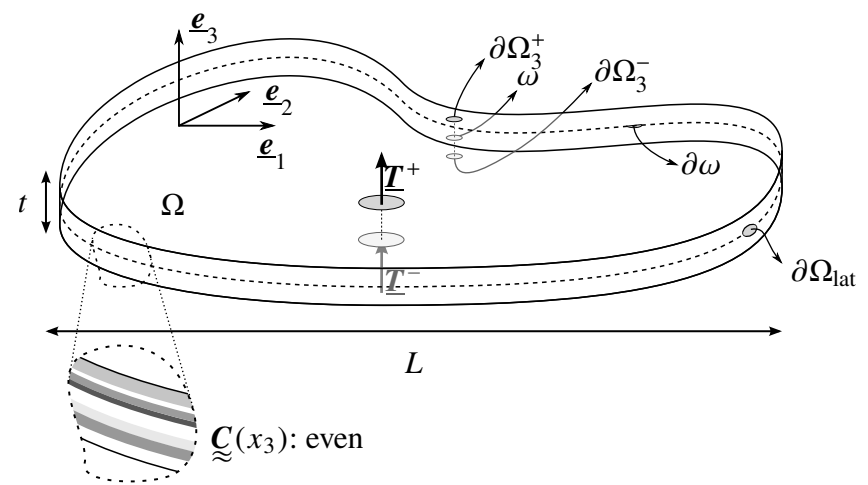

Fig. 1 The 3D configuration

In the following, we assume that there are no body forces and that the plate is fully clamped on its lateral boundary $\partial \Omega_{\text {lat }}$.

The fourth-rank $3 D$ elasticity stiffness tensor $\left(C_{i j k l}\right)$ has both the minor and major symmetries $C_{i j k l}=C_{j i k l}=C_{k l i j}$ and it is positive definite. Its inverse is called the $3 D$ elastic compliance tensor and is denoted by $\left(S_{i j k l}\right)$. The tensor $\left(S_{i j k l}\right)$ possesses the same symmetries as $\left(C_{i j k l}\right)$ and it is also positive definite.

We suppose that the constitutive material is invariant with respect to translations in the $\left(x_{1}, x_{2}\right)$ plane. Therefore, $C_{i j k l}$ does not depend on $\left(x_{1}, x_{2}\right)$ and is an even function of $x_{3}$ :

$$
C_{i j k l}\left(x_{3}\right)=C_{i j k l}\left(-x_{3}\right) \text {. }
$$

Furthermore, we have that:

$$
C_{3 \alpha \beta \gamma}=C_{\alpha 333}=0 .
$$

In this case, the constitutive material is said to be monoclinic.

The following notations are needed for the partial compliance tensors:

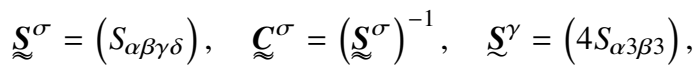


where $\underset{\sim}{\boldsymbol{S}}{ }^{\sigma}$ corresponds to plane stress compliance, $\underset{\sim}{\boldsymbol{C}}$ to plane stress stiffness and ${\underset{\sim}{\boldsymbol{S}}}^{\gamma}$ to transverse shear compliance.

The $3 D$ elastic problem is to find in $\Omega$ a displacement field $\underline{\boldsymbol{u}}$, a stress tensor field $\underset{\sim}{\sigma}$ and a strain tensor field $\underset{\mathcal{\varepsilon}}{\boldsymbol{E}}$, solution of the following equations:

$$
\left\{\begin{array}{l}
\underset{\sim}{\boldsymbol{\sigma}} \cdot \underline{\boldsymbol{\nabla}}=0, \quad \text { on } \quad \Omega, \\
\underset{\sim}{\boldsymbol{\sigma}}=\underset{\sim}{\boldsymbol{C}}\left(x_{3}\right): \boldsymbol{\varepsilon}, \quad \text { on } \Omega, \\
\boldsymbol{\sigma} \cdot \pm \underline{\boldsymbol{e}}_{3}=\underline{\boldsymbol{T}}^{ \pm}, \quad \text { on } \partial \Omega_{3}{ }^{ \pm}, \\
\underset{\boldsymbol{\varepsilon}}{=}=\frac{1}{2}(\underline{\boldsymbol{u}} \otimes \underline{\boldsymbol{\nabla}}+\underline{\boldsymbol{\nabla}} \otimes \underline{\boldsymbol{u}}), \quad \text { on } \quad \Omega, \\
\underline{\boldsymbol{u}}=0, \quad \text { on } \quad \partial \Omega_{\text {lat }} .
\end{array}\right.
$$

\subsection{The Bending-Gradient theory}

We now introduce the main definitions and equations of the Bending-Gradient theory, which main purpose is to replace the full $3 D$ model by a reduced $2 D$ plate theory and to be able to reconstruct the $3 \mathrm{D}$ solution fields from the $2 D$ solution fields.

Following the ideas of [30] for homogeneous plates, Lebée and Sab [13] derived a new plate theory, called the Bending-Gradient theory, suitable for heterogeneous plates. In this theory, the full gradient of the bending moment is considered as a generalized shear stress. Note that this theory cannot be reduced to a Reissner-Mindlin theory in the general case. However, it is turned into a Reissner-Mindlin theory when the plate under consideration is homogeneous (see [32, 19, 20] for more details). This shift between theories is directly related to the positive definiteness of the generalized shear constitutive tensor defined in the following. In this section and up to Section 7 it is assumed that the generalized shear constitutive tensor is positive definite. Section 7 will treat the case when the definiteness is lost.

\subsubsection{Generalized stresses}

The Bending-Gradient theory generalized stresses are:

- The bending moment tensor $\underset{\sim}{\boldsymbol{M}}$ related to the $3 D$ local stress $\left(\sigma_{i j}\right)$ by:

$$
\underset{\sim}{\boldsymbol{M}}=\left(M_{\alpha \beta}\right)=\left(\left\langle x_{3} \sigma_{\alpha \beta}\right\rangle\right),
$$

where the integration through the thickness is noted $\langle\bullet\rangle: \int_{-\frac{t}{2}}^{\frac{t}{2}} f\left(x_{3}\right) d x_{3}=\langle f\rangle$. We keep in mind that the second-order tensor $\underset{\sim}{\boldsymbol{M}}$ has the following symmetry:

$$
M_{\alpha \beta}=M_{\beta \alpha} .
$$

- The generalized shear force, denoted by $\underline{R}$ and defined as the gradient of the bending moment:

$$
\underline{\boldsymbol{R}}=\underline{\boldsymbol{M}} \otimes \underline{\boldsymbol{\nabla}} .
$$

In terms of components, this relation is written as $R_{\alpha \beta \gamma}=M_{\alpha \beta, \gamma}$. Note that the $2 \mathrm{D}$ third-order tensor $R_{\alpha \beta \gamma}$ complies with the following symmetry:

$$
R_{\alpha \beta \gamma}=R_{\beta \alpha \gamma} .
$$


Remark 1 The usual transverse shear force $\underline{Q}$ is defined from the $3 D$ stress field $\sigma$ as follows:

$$
\underline{\underline{Q}}=\left(Q_{\alpha}\right)=\left(\left\langle\sigma_{\alpha 3}\right\rangle\right) \text {. }
$$

It is interesting to note that the $3 D$ equilibrium equations enforce the following relation between $\underline{Q}$ and $\underline{R}$ :

$$
\underline{Q}=\underset{\sim}{\boldsymbol{i}}: \underline{\boldsymbol{R}}=\underset{\sim}{\boldsymbol{M}} \cdot \underline{\nabla}
$$

which can be written in terms of components as:

$$
Q_{\alpha}=R_{\alpha \beta \beta}=M_{\alpha \beta, \beta} .
$$

\subsubsection{Generalized displacements}

The Bending-Gradient generalized displacements are $(W, \boldsymbol{\Phi})$. The scalar $W$ is called the outof-plane displacement of the plate (or deflection) and $\boldsymbol{\Phi}$ is the generalized rotation tensor. The $2 D$ third-order tensor $\boldsymbol{\Phi}$ complies with the following symmetry:

$$
\Phi_{\alpha \beta \gamma}=\Phi_{\beta \alpha \gamma} .
$$

These displacements are interpreted physically as suitable averages of the $3 D$ displacements over the thickness of the plate. The reader is referred to [32] (Chapter 5) for corresponding details.

The Bending-Gradient generalized strains, which derive from $(W, \Phi)$, and constitute the dual of the generalized stresses $(\underset{\sim}{\boldsymbol{M}}, \underline{\boldsymbol{R}})$ are:

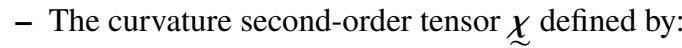

$$
\underset{\sim}{\chi}=\Phi \cdot \underline{\boldsymbol{\nabla}}, \quad \text { or } \quad \chi_{\alpha \beta}=\Phi_{\alpha \beta \gamma, \gamma} .
$$

- The generalized shear strain $\underset{\boldsymbol{\Gamma}}{\text { given by: }}$

$$
\underline{\boldsymbol{\Gamma}}=\underline{\boldsymbol{\Phi}}+\underset{\approx}{\boldsymbol{i}} \cdot \underline{\boldsymbol{\nabla}} W, \quad \text { or } \quad \Gamma_{\alpha \beta \gamma}=\Phi_{\alpha \beta \gamma}+i_{\alpha \beta \gamma \delta} W_{, \delta} .
$$

$\boldsymbol{\Gamma}$ is a third-order $2 \mathrm{D}$ tensor which comply with the following symmetry:

$$
\Gamma_{\alpha \beta \gamma}=\Gamma_{\beta \alpha \gamma} .
$$

Equations 28) and 29) are called the compatibility conditions on $\omega$.

In the following section, we assume clamped boundary conditions. Nevertheless, various boundary conditions (free, simply supported..) are also considered in this paper (see Sects. 5 and 6 . The clamped boundary conditions on $\partial \omega$ read:

$$
\underline{\Phi} \cdot \underline{\boldsymbol{n}}=0, \quad \text { and } \quad W=0, \quad \text { on } \partial \omega,
$$

where $\underline{\boldsymbol{n}}$ is the outer normal vector to $\partial \omega$.

Note that the kinematics of the Bending-Gradient theory coincides with the ReissnerMindlin kinematics if the deviatoric part of $\Phi$ is set to zero. We can hence interpret the Reissner-Mindlin kinematics as the restriction of $\underline{\Phi}$ to $\underset{\sim}{\boldsymbol{i}} \cdot \underline{\varphi}$, where $\underline{\varphi}$ designates a rotation vector. 


\subsubsection{Equilibrium equations}

We start by recalling that integrating the $3 D$ equilibrium equation $25 \mathrm{a}$ ) leads to the wellknown Reissner-Mindlin plate equilibrium equations:

$$
\left\{\begin{array}{l}
\underset{\boldsymbol{M}}{\tilde{\boldsymbol{Q}}} \cdot \underline{\boldsymbol{\nabla}}=\underline{\boldsymbol{Q}}, \\
\underline{\boldsymbol{\nabla}}+p=0 .
\end{array}\right.
$$

Considering that $(\underset{\sim}{\boldsymbol{M}}, \underline{\boldsymbol{R}})$ are the generalized stresses of the Bending-Gradient theory instead of $(\underline{\boldsymbol{M}}, \underline{\boldsymbol{Q}})$ for the Reissner-Mindlin theory, the above equilibrium equations become:

$$
\left\{\begin{array}{l}
\underline{\boldsymbol{R}}-\underset{\boldsymbol{M}}{ } \otimes \underline{\boldsymbol{\nabla}}=0, \\
\underset{\sim}{\boldsymbol{i}} \vdots(\underline{\boldsymbol{R}} \otimes \underline{\boldsymbol{\nabla}})+p=0,
\end{array}\right.
$$

in the case when the generalized shear constitutive tensor is positive definite. Otherwise, the modified equilibrium equations are defined in Section 7

Note that using the first equilibrium equation to eliminate $\underline{R}$ in the second equilibrium equation gives the equivalent form:

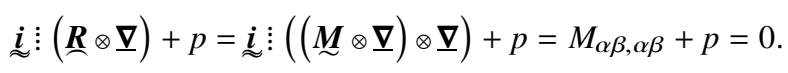

\subsubsection{Constitutive equations}

Finally, the Bending-Gradient constitutive equations write:

$$
\left\{\begin{array}{l}
\underset{\sim}{\chi}=\underset{\sim}{\boldsymbol{d}}: \underset{\sim}{\boldsymbol{M}}, \\
\underline{\boldsymbol{\Gamma}}=\underline{\boldsymbol{h}}: \underline{\boldsymbol{R}} .
\end{array}\right.
$$

Here, $\underset{\sim}{\boldsymbol{d}}$ is the classical bending compliance fourth-order tensor, inverse of the bending stiffness fourth-order tensor $\underset{\sim}{\mathbb{D}}$. It is given by:

$$
\underset{\approx}{\boldsymbol{d}}={\underset{\approx}{\boldsymbol{D}}}^{-1}, \quad \text { with } \underset{\approx}{\boldsymbol{D}}=\left\langle x_{3}^{2}{\underset{\approx}{\boldsymbol{C}}}^{\sigma}\right\rangle
$$

Tensors $\underset{\approx}{\boldsymbol{d}}$ and $\underset{\approx}{\boldsymbol{D}}$ are symmetric positive and definite. They follow both major symmetry ${ }^{T} \underset{\approx}{\boldsymbol{D}}=\underset{\approx}{\boldsymbol{D}}$ and minor symmetry $D_{\alpha \beta \gamma \delta}=D_{\beta \alpha \gamma \delta}$.

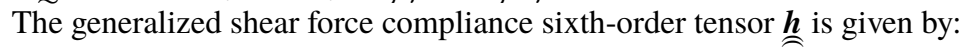

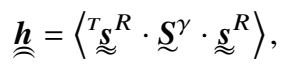

where the fourth order tensor ${\underset{\approx}{s}}^{R}\left(x_{3}\right)$ is the function of $x_{3}$ defined by:

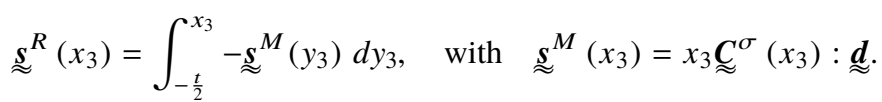

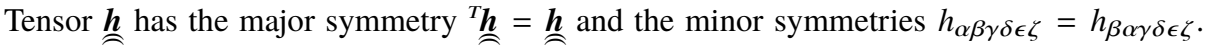
It is positive but not always definite. Three particular cases should be distinguished when dealing with $\underset{\boldsymbol{h}}{\boldsymbol{h}}$ :

- The first one is when the sixth-order tensor is positive definite. In this case, its inverse is

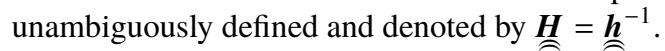


- The second case is when the shear force compliance tensor can be written in the following form:

$$
\stackrel{\boldsymbol{h}}{\approx} \underset{\approx}{\boldsymbol{i}} \cdot \underline{\boldsymbol{h}}^{\mathrm{RM}} \cdot \underset{\sim}{\boldsymbol{i}}
$$

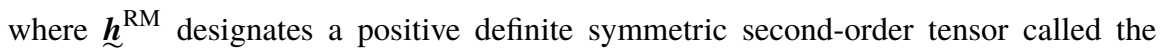
Reissner-Mindlin shear force compliance tensor. In this case, the Bending-Gradient theory degenerates into a Reissner-Mindlin theory. The existence and uniqueness of the solution in this case is already established in the literature.

- The third case is an intermediate between the above mentioned cases. It will be studied in details in Section 7

In the following (except in Section 7), it will be assumed that the sixth-order tensor $\boldsymbol{h}$ is positive definite. The Bending-Gradient constitutive equations can thus be inverted:

$$
\left\{\begin{array}{l}
\underset{\sim}{\boldsymbol{M}}=\underset{\sim}{\boldsymbol{D}}: \underset{\sim}{\boldsymbol{\chi}}, \\
\underline{\boldsymbol{R}}=\underset{\sim}{\boldsymbol{H}}: \underline{\boldsymbol{\Gamma}} .
\end{array}\right.
$$

\subsubsection{Summary of the Bending-Gradient plate theory}

Finally, the Bending-Gradient problem enables a good approximation of the 3D fields introduced earlier including the effects of transverse shear. It consists in finding the generalized displacement $(W, \Phi)$ solution of the following equations:

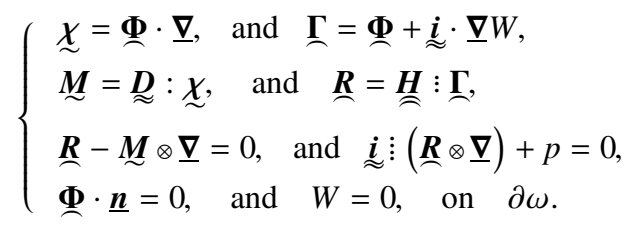

It should be emphasized that once we find the solution of the Bending-Gradient problem, we can reconstruct the $3 D$ stress and displacement fields (see Chapter 5 of [32] for more details).

Now that we have presented the main features of the Bending-Gradient theory, it is useful to recall its variational approach as stated in [32]. It has to be pointed out first that generalized displacements $(W, \Phi)$ which satisfy the boundary conditions 38 d) are called kinematically compatible fields. Besides, generalized stresses $(\underset{\sim}{\boldsymbol{M}}, \boldsymbol{R})$ that comply with equations $38 \mathrm{c})$ are designated as statically compatible fields.

\subsubsection{Minimum of the potential energy}

The principle of the minimum of the potential energy states that the solution $\left(W^{\mathrm{BG}}, \boldsymbol{\Phi}^{\mathrm{BG}}\right)$ of the Bending-Gradient problem (38) renders the potential energy functional $\mathcal{E}^{\mathrm{BG}}$ a minimum value on the set of kinematically compatible Bending-Gradient displacements. Here, the potential energy $\mathcal{E}^{\mathrm{BG}}$ is defined as:

$$
\mathcal{E}^{\mathrm{BG}}=\int_{\omega} w^{\mathrm{BG}}(\underset{\sim}{\boldsymbol{\chi}}, \boldsymbol{\Gamma})-\int_{\omega} p W
$$


where $\underset{\sim}{\boldsymbol{\chi}}$ and $\boldsymbol{\Gamma}$ are the generalized strains related to the generalized displacements $(W, \boldsymbol{\Phi})$ through the compatibility equations 38 a). Furthermore, $w^{\mathrm{BG}}$ is the Bending-Gradient strain energy density function given by:

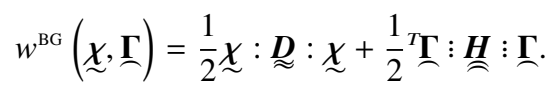

\subsubsection{Minimum of the complementary energy}

Similarly, we introduce the complementary energy $\mathcal{E}^{* \mathrm{BG}}$ of the Bending-Gradient problem which is defined as:

$$
\mathcal{E}^{* \mathrm{BG}}=\int_{\omega} w^{* \mathrm{BG}}(\underset{\sim}{\boldsymbol{M}}, \underset{\boldsymbol{R}}{)},
$$

where $w^{* \mathrm{BG}}$ represents the Bending-Gradient stress energy density function given by:

$$
w^{* \mathrm{BG}}(\underset{\sim}{\boldsymbol{M}}, \underline{\boldsymbol{R}})=\frac{1}{2} \underset{\sim}{\boldsymbol{M}}: \underset{\sim}{\boldsymbol{d}}: \underset{\sim}{\boldsymbol{M}}+\frac{1}{2}^{T} \underline{\boldsymbol{R}}: \underset{\sim}{\boldsymbol{h}}: \underline{\boldsymbol{R}} .
$$

The principle of minimum complementary energy states that, among all statically compatible stress fields, the complementary energy functional $\mathcal{E}^{* \mathrm{BG}}$ assumes a minimum value for the stress field $\left({\underset{\boldsymbol{M}}{\boldsymbol{B G}}}^{\mathrm{BG}}, \underline{\boldsymbol{R}}^{\mathrm{BG}}\right)$, solution of the Bending-Gradient problem.

Having set the Bending-Gradient problem, our main concern now is to define the functional spaces in which we shall seek solutions and introduce norms in order to show that the corresponding variational problems, namely the stress and the displacement formulations, are well-posed.

\section{Mathematical formulation of the Bending-Gradient problem for a fully clamped plate}

We study in this section both stress and displacement formulations of the Bending-Gradient theory presented in the previous section and show their well-posedness.

\subsection{Stress formulation}

It is based on the principle of the minimum of the complementary energy (Section 3.2.7). Since the bending moment tensor $\underset{\sim}{\boldsymbol{M}}$ and the generalized shear force $\boldsymbol{R}$ are related by the equilibrium equation (32), the complementary energy $\mathcal{E}^{* \mathrm{BG}}$ will be expressed solely in terms of $\boldsymbol{M}$. Therefore, it can be equivalently written in the following form:

$$
\mathcal{E}^{* \mathrm{BG}}(\underset{\sim}{\boldsymbol{M}})=\int_{\omega} w^{* \mathrm{BG}}(\underset{\sim}{\boldsymbol{M}}, \underset{\sim}{\boldsymbol{M}} \otimes \underline{\boldsymbol{\nabla}}),
$$

where $w^{* \mathrm{BG}}$ is defined by 42 .

We recall that the compliance tensors $\underset{\widetilde{d}}{\boldsymbol{d}}$ and $\underset{\boldsymbol{h}}{\boldsymbol{h}}$ are positive definite in the sense that there exists two constants $c>0$ and $c^{\prime}>0$ such that:

$$
\begin{aligned}
& \forall \underset{\sim}{\boldsymbol{M}} \in \underset{\sim}{\mathbb{R}}, \quad c \underset{\sim}{\boldsymbol{M}}: \underset{\sim}{\boldsymbol{M}} \leq \underset{\sim}{\boldsymbol{M}}: \underset{\sim}{\boldsymbol{d}}: \underset{\sim}{\boldsymbol{M}} \leq c^{\prime} \underset{\sim}{\boldsymbol{M}}: \underset{\sim}{\boldsymbol{M}},
\end{aligned}
$$

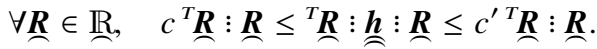


Note that relations (44) and 45) also hold for the stiffness tensors $\underset{\sim}{\boldsymbol{D}}$ and $\boldsymbol{H}$.

Since the generalized stresses $(\boldsymbol{M}, \underline{\boldsymbol{R}})$ that comply with equations 38 c) are such that:

$$
\underset{\sim}{i} \vdots(\underline{\boldsymbol{R}} \otimes \underline{\boldsymbol{\nabla}})=\underset{\sim}{\boldsymbol{i}} \vdots((\underline{\boldsymbol{M}} \otimes \underline{\boldsymbol{\nabla}}) \otimes \underline{\boldsymbol{\nabla}})=(\underline{\boldsymbol{M}} \cdot \underline{\boldsymbol{\nabla}}) \cdot \underline{\boldsymbol{\nabla}}=M_{\alpha \beta, \alpha \beta},
$$

then it is natural to define the space of statically admissible generalized stress fields as follows:

$$
\operatorname{SC}(p)=\left\{\underset{\sim}{\boldsymbol{M}} \in{\underset{\sim}{\boldsymbol{H}}}^{1}(\omega), \quad M_{\alpha \beta, \alpha \beta}+p=0 \text { in the sense of distributions }\right\} .
$$

The variational formulation of the Bending-Gradient problem consists in minimizing the complementary energy $\mathcal{E}^{* \mathrm{BG}}(\underset{\sim}{\boldsymbol{M}})$ with respect to all $\underset{\sim}{\boldsymbol{M}} \in \operatorname{SC}(p)$ :

$$
\min \left\{\mathcal{E}^{* \mathrm{BG}}(\underset{\sim}{\boldsymbol{M}})=\int_{\omega} w^{* \mathrm{BG}}(\underset{\sim}{\boldsymbol{M}}, \underset{\sim}{\boldsymbol{M}} \otimes \underline{\nabla}), \quad \underset{\sim}{\boldsymbol{M}} \in \operatorname{SC}(p)\right\} .
$$

4.2 Well-posedness of the stress formulation

We now proceed to the study of problem (47). We establish the following result:

Theorem 1 Assume that $p \in H^{-1}(\omega)$ and that assumptions (44) and (45) hold. Then, the minimization problem 47) admits a unique solution $\boldsymbol{M}^{\mathrm{BG}} \in \mathrm{SC}(p)$. Moreover, $\boldsymbol{M}^{\mathrm{BG}}$ is solution of the following variational problem:

$$
\left\{\begin{array}{l}
\text { Find } \boldsymbol{M}^{\mathrm{BG}} \in \operatorname{SC}(p), \text { such that: } \\
\int_{\omega}^{\boldsymbol{\mu}}: \underset{\sim}{\boldsymbol{d}}:{\underset{\sim}{\boldsymbol{M}}}^{\mathrm{BG}}+(\underset{\sim}{\boldsymbol{\mu}} \otimes \underline{\boldsymbol{\nabla}}): \underset{\sim}{\boldsymbol{h}}:\left({\underset{\sim}{\boldsymbol{M G}}}^{\mathrm{BG}} \underline{\boldsymbol{\nabla}}\right)=0, \quad \forall \underset{\sim}{\boldsymbol{\mu}} \in \operatorname{SC}(0) .
\end{array}\right.
$$

Proof Let us first show that $\operatorname{SC}(p)$ is not empty. Indeed, let $m$ be the unique solution in $H_{0}^{1}(\omega)$ of equation $\Delta m+p=0$. Then, $\underset{\sim}{\boldsymbol{P}}$ defined by $P_{\alpha \beta}=m \delta_{\alpha \beta}$ is obviously in $\operatorname{SC}(p)$. Moreover, any $\underset{\sim}{\boldsymbol{M}} \in S C(p)$ can be decomposed as the sum of $\underset{\sim}{\boldsymbol{P}}$ and $\underset{\sim}{\boldsymbol{\mu}}$ in $S C(0)$. Namely:

$$
\underset{\sim}{\boldsymbol{M}} \in \operatorname{SC}(p) \Longleftrightarrow \underset{\sim}{\boldsymbol{M}}=\underset{\sim}{\boldsymbol{P}}+\underset{\sim}{\boldsymbol{\mu}}, \quad \underset{\sim}{\boldsymbol{\mu}} \in \operatorname{SC}(0) .
$$

We introduce the following symmetric bilinear form on ${\underset{\sim}{\boldsymbol{H}}}^{1}(\omega) \times{\underset{\sim}{\boldsymbol{H}}}^{1}(\omega)$ :

$$
a_{s}\left(\underset{\sim}{\boldsymbol{M}}, \underline{\boldsymbol{M}}^{\prime}\right)=\int_{\omega} \boldsymbol{M}^{\prime}: \underset{\sim}{\boldsymbol{d}}: \underset{\sim}{\boldsymbol{M}}+{ }^{T}\left(\underline{\boldsymbol{M}}^{\prime} \otimes \underline{\boldsymbol{\nabla}}\right): \underset{\sim}{\boldsymbol{h}}:(\underset{\sim}{\boldsymbol{M}} \otimes \underline{\boldsymbol{\nabla}}),
$$

which enables us to write $\mathcal{E}^{* \mathrm{BG}}$ as:

$$
\mathcal{E}^{* \mathrm{BG}}(\underset{\sim}{\boldsymbol{M}})=\frac{1}{2} a_{s}(\underset{\sim}{\boldsymbol{M}}, \underset{\sim}{\boldsymbol{M}}) .
$$

Using the decomposition 49, it can be easily seen that the following minimization problem:

$$
\min \left\{\frac{1}{2} a_{S}(\underset{\sim}{\boldsymbol{\mu}} \underset{\sim}{\boldsymbol{\mu}})+a_{S}(\underset{\sim}{\boldsymbol{P}} \underset{\sim}{\boldsymbol{\mu}}), \quad \underset{\sim}{\boldsymbol{\mu}} \in \operatorname{SC}(0)\right\},
$$

is equivalent to problem 47 . 
The space $S C(0)$ equipped with the scalar product $\langle., .\rangle_{S C(0)}$ is actually a Hilbert space as shown in Appendix A.1.

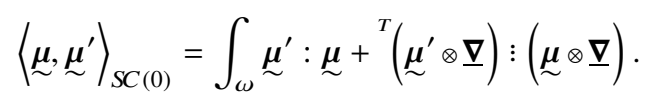

Clearly, thanks to assumptions 444 , and (45), the bilinear form $\left.a_{s}(\underset{\sim}{\mu} \underset{\sim}{\mu})^{\prime}\right)$ is continuous and coercive on $S C(0)$ and the linear map $\left.\underset{\sim}{\boldsymbol{\mu}} \longrightarrow a_{s} \underset{\sim}{\underset{\sim}{\boldsymbol{P}}} \underset{\sim}{\boldsymbol{\mu}}\right)$ is continuous on $S C(0)$. See Appendix A.2. Hence, by the application of the Lax-Milgram theorem on 50, we conclude that the

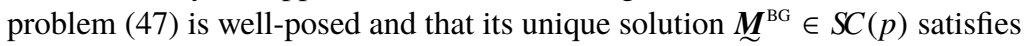

$$
\forall \underset{\sim}{\mu} \in \operatorname{SC}(0), \quad a_{S}\left(\underset{\sim}{\mu}, \underline{\sim}^{\mathrm{BG}}\right)=0
$$

This ends the proof.

4.3 Well-posedness of the displacement formulation

Let $(W, \Phi)$ be a generalized displacement field defined over $\omega$. The potential energy $\mathcal{E}^{\mathrm{BG}}$ expressed in terms of $(W, \Phi)$ takes the form:

$$
\mathcal{E}^{\mathrm{BG}}(W, \underline{\mathbf{\Phi}})=\int_{\omega} w^{\mathrm{BG}}(\underline{\boldsymbol{\Phi}} \cdot \underline{\boldsymbol{\nabla}}, \underline{\mathbf{\Phi}}+\underset{\sim}{\boldsymbol{i}} \cdot \underline{\boldsymbol{\nabla}} W)-\int_{\omega} p W,
$$

where $w^{\mathrm{BG}}$ is given by (40).

Assuming $p \in H^{-1}(\omega), \mathcal{E}^{\mathrm{BG}}$ is clearly well defined for $(W, \Phi) \in K C_{0}$ where the space of the generalized Bending-Gradient displacements which are kinematically compatible with clamped boundary conditions is the product of $H_{0}^{1}(\omega)$ with the space $\underline{\boldsymbol{H}}_{0}(\operatorname{div}, \omega)$ introduced in Section 2.2.

$$
K C_{0}=H_{0}^{1}(\omega) \times \underline{H}_{0}(\operatorname{div}, \omega) .
$$

Note that the space $K C_{0}$ is a Hilbert space as the product of two Hilbert spaces.

The Bending-Gradient problem with clamped boundary conditions consists in minimizing the potential energy $\mathcal{E}^{\mathrm{BG}}(W, \Phi)$ over all $(W, \Phi) \in K C_{0}$ :

$$
\min \left\{\mathcal{E}^{\mathrm{BG}}(W, \underline{\Phi}), \quad(W, \underline{\Phi}) \in K C_{0}\right\},
$$

where $\mathcal{E}^{\mathrm{BG}}$ is given by 51 .

In order to show that the minimization problem 52 is well-posed, we introduce the bilinear form $a$ defined on $K C_{0} \times K C_{0}$ by:

$a\left((W, \underline{\boldsymbol{\Phi}}),\left(W^{\prime}, \underline{\Phi}^{\prime}\right)\right)=\int_{\omega}(\underline{\boldsymbol{\Phi}} \cdot \underline{\boldsymbol{\nabla}}): \underset{\approx}{\boldsymbol{D}}:\left(\underline{\Phi}^{\prime} \cdot \underline{\boldsymbol{\nabla}}\right)+{ }^{T}(\underline{\boldsymbol{\Phi}}+\underset{\sim}{\boldsymbol{i}} \cdot \underline{\boldsymbol{\nabla}} W): \underline{\boldsymbol{H}}:\left(\underline{\boldsymbol{\Phi}}^{\prime}+\underset{\sim}{\boldsymbol{i}} \cdot \underline{\boldsymbol{\nabla}} W^{\prime}\right)$,

and the linear form $b$ defined on $K C_{0}$ by:

$$
b(W, \Phi)=\int_{\omega} p W .
$$


Hence, $\mathcal{E}^{\mathrm{BG}}(W, \Phi)$ can be written as:

$$
\mathcal{E}^{\mathrm{BG}}(W, \boldsymbol{\Phi})=\frac{1}{2} a((W, \underline{\Phi}),(W, \Phi))-b(W, \underline{\Phi}) .
$$

Clearly, the linear form $b$ is continuous on $K C_{0}$ (see Appendix A.3). Furthermore, the bilinear form $a$ is symmetric and continuous on $K C_{0} \times K C_{0}$ (see Appendix A.4). It thus remains to prove that $a$ is coercive on $K C_{0}$.

\subsubsection{Coercivity of the bilinear form a}

Let us introduce a new bilinear form denoted by $a_{N}$ and defined on $K C_{0} \times K C_{0}$ by:

$$
a_{N}\left((W, \underline{\boldsymbol{\Phi}}),\left(W^{\prime}, \underline{\Phi}^{\prime}\right)\right)=\int_{\omega}(\underline{\boldsymbol{\Phi}} \cdot \underline{\boldsymbol{\nabla}}):\left(\underline{\Phi}^{\prime} \cdot \underline{\boldsymbol{\nabla}}\right)+{ }^{T}(\underline{\boldsymbol{\Phi}}+\underset{\sim}{\boldsymbol{i}} \cdot \underline{\boldsymbol{\nabla}} W):\left(\underline{\boldsymbol{\Phi}}^{\prime}+\underset{\sim}{\boldsymbol{i}} \cdot \underline{\boldsymbol{\nabla}} W^{\prime}\right) .
$$

Since $\underset{\sim}{\boldsymbol{D}}$ and $\underset{\widetilde{\boldsymbol{H}}}{\widetilde{a}}$ are positive definite, there exists a constant $\beta>0$ such that, for all $\underset{\sim}{\boldsymbol{\chi}} \underset{\sim}{\mathbb{R}}$ and all $\boldsymbol{\Gamma} \in \underline{\mathbb{R}}$, we have:

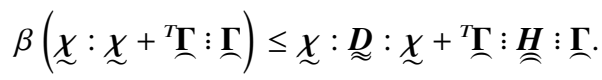

Hence, the coercivity of $a$ over $K C_{0} \times K C_{0}$ directly follows from the below lemma:

Lemma 6 The bilinear form $a_{N}$ is a scalar product on $K C_{0}$ and the associated norm denoted by $\|\bullet\|_{N}$ is equivalent to the norm $\|\bullet\|_{K C_{0}}$.

Proof We decompose the proof into two steps.

Step 1. Since the bilinear form $a_{N}$ is symmetric and positive, it only remains to prove that it is definite on $K C_{0}$ in order to show that it is a scalar product. Consider $(W, \Phi) \in K C_{0}$ such that:

$$
a_{N}((W, \Phi),(W, \Phi))=0 .
$$

Then, $\underline{\boldsymbol{\Phi}} \cdot \underline{\boldsymbol{\nabla}}=0$ and $\underline{\boldsymbol{\Phi}}+\underset{\sim}{\boldsymbol{i}} \cdot \underline{\boldsymbol{\nabla}} W=0$. We therefore have that $\Delta W=0$. This implies that $W=0$, as $W \in H_{0}^{1}(\omega)$. Hence, $\boldsymbol{\Phi}=0$. Thus, $a_{N}$ is a scalar product on $K C_{0}$ and $\|\bullet\|_{N}$ defines a norm on $K C_{0}$.

Step 2. It is important to note that there exists a constant $C$ such that:

$$
\begin{aligned}
& \forall(W, \underline{\Phi}) \in K C_{0}, \quad\|W, \underline{\boldsymbol{\Phi}}\|_{N}^{2}=\|\underline{\boldsymbol{\Phi}} \cdot \underline{\boldsymbol{\nabla}}\|_{\underline{\boldsymbol{L}}^{2}(\omega)}^{2}+\|\underline{\boldsymbol{\Phi}}+\underset{\sim}{\boldsymbol{i}} \cdot \underline{\boldsymbol{\nabla}} W\|_{\underline{\mathbf{L}}^{2}(\omega)}^{2} \\
& \leq C\|W, \Phi\|_{K C_{0}}^{2} \text {. }
\end{aligned}
$$

In order to show that the norms $\|\cdot, \cdot\|_{N}$ and $\|\cdot, \cdot\|_{K C_{0}}$ are equivalent, we need to prove that there exists a constant $C^{\prime}$ such that :

$$
\forall(W, \Phi) \in K C_{0}, \quad\|W, \Phi\|_{K C_{0}} \leq C^{\prime}\|W, \Phi\|_{N} .
$$

To this aim, we proceed by contradiction. Suppose that for all $k \in \mathbb{N}$, there exists $\left(W_{k}, \Phi_{k}\right) \in K C_{0}$ such that:

$$
\left\|W_{k}, \Phi_{k}\right\|_{K C_{0}}=1, \quad\left\|W_{k}, \Phi_{k}\right\|_{N} \leq \frac{1}{k} .
$$


Rather than working with the third order tensor $\Phi$, it is more useful to work with its spherical part $\Phi^{\mathrm{s}}$ and its deviatoric part $\Phi^{\mathrm{d}}$. We define the spherical part $\Phi^{\mathrm{s}}$ by:

$$
\underline{\Phi}^{\mathrm{s}}=\underset{\sim}{\boldsymbol{i}} \cdot \underline{\Phi}^{\mathrm{s}}, \quad \underline{\Phi}^{\mathrm{s}}=\frac{2}{3} \Phi: \underset{\sim}{\boldsymbol{\delta}}
$$

where $\underline{\boldsymbol{\Phi}}^{\mathrm{s}}$ is in $\underline{\boldsymbol{L}}^{2}(\omega)$. We can therefore write $\left\|W_{k}, \underline{\boldsymbol{\Phi}}_{k}\right\|_{N}^{2}$ as follows:

$$
\begin{aligned}
& \left\|W_{k}, \underline{\Phi}_{k}\right\|_{N}^{2}=\left\|\underline{\Phi}_{k} \cdot \underline{\boldsymbol{\nabla}}\right\|_{\underline{L}^{2}(\omega)}^{2}+\left\|\underline{\Phi}_{k}^{\mathrm{d}}+\underline{\boldsymbol{\Phi}}_{k}^{\mathrm{s}}+\underset{\sim}{\boldsymbol{i}} \cdot \underline{\boldsymbol{\nabla}} W_{k}\right\|_{\underline{L}^{2}(\omega)}^{2} \\
& =\left\|\underline{\Phi}_{k} \cdot \underline{\boldsymbol{\nabla}}\right\|_{\underline{\underline{L}}^{2}(\omega)}^{2}+\left\|\underline{\boldsymbol{\Phi}}_{k}^{\mathrm{d}}\right\|_{\underline{\mathbf{L}}^{2}(\omega)}^{2}+\frac{3}{2}\left\|\underline{\boldsymbol{\Phi}}_{k}^{\mathrm{s}}+\underline{\boldsymbol{\nabla}} W_{k}\right\|_{\underline{\mathbf{L}}^{2}(\omega)}^{2} .
\end{aligned}
$$

Using (58), we have that $\left\|\Phi_{k} \cdot \underline{\nabla}\right\|_{\underline{L}^{2}(\omega)}+\left\|\Phi_{k}^{\mathrm{d}}\right\|_{\underline{\boldsymbol{L}}^{2}(\omega)} \leq \frac{1}{k}$. It follows from Lemma 3 that $\left(\stackrel{\Phi}{k}_{k}\right)$ strongly converges to zero in $\boldsymbol{H}(\operatorname{div}, \omega)$. Using [59], we can assure that $\underline{\boldsymbol{\Phi}}_{k}^{\text {s }}$ strongly converges to zero in $\underline{\boldsymbol{L}}^{2}(\omega)$.

Hence,

$$
\left\|\underline{\Phi}_{k}^{\mathrm{s}}+\underline{\boldsymbol{\nabla}} W_{k}\right\|_{\underline{\boldsymbol{L}}^{2}(\omega)} \leq \frac{1}{k}
$$

implies that $\left(\underline{\nabla} W_{k}\right)$ strongly converges to zero in $\underline{\boldsymbol{L}}^{2}(\omega)$. Consequently, $\left(W_{k}\right)$ strongly converges to zero in $H_{0}^{1}(\omega)$. There is hence in contradiction with $\left\|W_{k}, \Phi_{k}\right\|_{K C_{0}}=1$.

\subsubsection{Existence and uniqueness of the solution to (52)}

The existence and uniqueness of the solution to the minimization problem (52) is directly inferred from the Lax-Milgram theorem since its assumptions are satisfied. Namely, the symmetric bilinear form $a$ is continuous and coercive, and the linear form $b$ is continuous. The main result of this paper is stated in the following theorem:

Theorem 2 Assume that $p \in H^{-1}(\omega)$ and that the stiffness tensors $\underset{\widetilde{D}}{\mathbf{D}}$ and $\underset{\widetilde{H}}{\boldsymbol{H}}$ are symmetric and positive definite. Then the problem (52) is well-posed, i.e., there exists a unique solution $\left(W^{\mathrm{BG}}, \Phi^{\mathrm{BG}}\right) \in K C_{0}$ such that:

$$
a\left(\left(W^{\mathrm{BG}}, \underline{\boldsymbol{\Phi}}^{\mathrm{BG}}\right),(W, \underline{\mathbf{\Phi}})\right)=b(W, \underline{\mathbf{\Phi}}),
$$

for all $(W, \Phi) \in K C_{0}$, where $a$ and $b$ are given by 53 and 54 . Moreover, we have

$$
\left\|W^{\mathrm{BG}}, \Phi^{\mathrm{BG}}\right\|_{K C_{0}} \leq c\|p\|_{H^{-1}(\omega)},
$$

where $c$ is a strictly positive constant. 
4.4 Relation between the stress and the displacement formulations

The above-presented formulations, namely the stress formulation (47) and the displacement formulation (52), have each respectively a unique solution, as shown respectively by Theorems 1 and 2 We therefore proceed to show that we can derive the displacement solution from the stress solution, and vice-versa. We state this in the following theorem:

Theorem 3 Under the assumptions stated in Theorem 2. let $\left(W^{\mathrm{BG}}, \boldsymbol{\Phi}^{\mathrm{BG}}\right)$ be the unique solution to the minimization problem (52). Consider that the generalized strains associated to $\left(W^{\mathrm{BG}}, \Phi^{\mathrm{BG}}\right)$ through the compatibility conditions $\sqrt{28},, 29$ ) are given by :

$$
\underline{\sim}^{\mathrm{BG}}=\underline{\boldsymbol{\Phi}}^{\mathrm{BG}} \cdot \underline{\boldsymbol{\nabla}}, \quad \underline{\boldsymbol{\Gamma}}^{\mathrm{BG}}=\underline{\boldsymbol{\Phi}}^{\mathrm{BG}}+\underset{\sim}{\boldsymbol{i}} \cdot \underline{\boldsymbol{\nabla}} W^{\mathrm{BG}},
$$

and that the generalized stress fields associated to $\left(W^{\mathrm{BG}}, \Phi^{\mathrm{BG}}\right)$ by the constitutive equations (33) are :

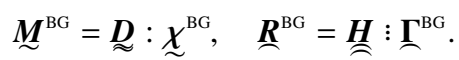

Then,

$$
{\underset{\sim}{\mathrm{BG}}}^{\mathrm{BG}} \in \operatorname{SC}(p),
$$

where $\operatorname{SC}(p)$ is defined by (46), and

$$
\underline{\boldsymbol{R}}^{\mathrm{BG}}=\underline{\boldsymbol{M}}^{\mathrm{BG}} \otimes \underline{\boldsymbol{\nabla}} .
$$

Moreover, $\boldsymbol{M}^{\mathrm{BG}}$ is the unique solution to the minimization problem $(47)$, where $w^{* \mathrm{BG}}$ is defined by (42).

Proof Since $\left(W^{\mathrm{BG}},{\underset{\boldsymbol{\Phi}}{\mathrm{BG}}}^{\mathrm{Bg}} \in K C_{0}\right.$, relations $\sqrt{63}$ yield ${\underset{\sim}{\boldsymbol{\chi}}}^{\mathrm{BG}} \in{\underset{\sim}{\boldsymbol{L}}}^{2}(\omega)$ and $\underline{\boldsymbol{\Gamma}}^{\mathrm{BG}} \in \underline{\boldsymbol{L}}^{2}(\omega)$. Consequently, due to equations $\sqrt{64}$, we also have that ${\underset{\sim}{\boldsymbol{M}}}^{\mathrm{BG}} \in \underline{\sim}^{2}(\omega)$ and $\underline{\boldsymbol{R}}^{\mathrm{BG}} \in \underline{\boldsymbol{L}}^{2}(\omega)$. According to theorem 2. $\left(W^{\mathrm{BG}}, \stackrel{\Phi}{\mathrm{BG}}\right)^{\mathrm{Batisfies}} 61$. Therefore, we have that, for all $(W, \underline{\Phi}) \in$ $K C_{0}$ :

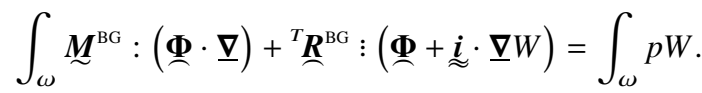

Restricting 66 to $(W, \Phi) \in D(\omega) \times \underset{D}{\boldsymbol{D}}(\omega) \subset K C_{0}$ and considering first the particular case where $W=$ yields after integration by parts:

$$
\left\langle\underline{\boldsymbol{R}}^{\mathrm{BG}}-\underline{\sim}^{\mathrm{BG}} \otimes \underline{\boldsymbol{\nabla}}, \underline{\boldsymbol{\Phi}}\right\rangle_{\underline{\boldsymbol{D}}^{\prime}(\omega), \underline{\boldsymbol{D}}(\omega)}=0 .
$$

This proves

$$
\underline{\boldsymbol{R}}^{\mathrm{BG}}=\underline{\boldsymbol{M}}^{\mathrm{BG}} \otimes \underline{\boldsymbol{\nabla}},
$$

in the sense of distributions.

Considering now the particular case where $\boldsymbol{\Phi}=0$ yields:

$$
\left\langle-\left(\underset{\sim}{\boldsymbol{i}}: \underline{\boldsymbol{R}}^{\mathrm{BG}}\right) \cdot \underline{\boldsymbol{\nabla}}-p, W\right\rangle_{D^{\prime}(\omega), D(\omega)}=0 .
$$

Hence,

$$
(\underset{\sim}{\boldsymbol{i}}: \underbrace{\mathrm{BG}}) \cdot \underline{\nabla}+p=0
$$

in the sense of distributions. 
Equation (68) shows that the bending gradient components are in $\underline{L}^{2}(\omega)$. Hence, $\boldsymbol{M}^{\mathrm{BG}} \in \stackrel{\boldsymbol{H}}{ }^{1}(\omega)$. Replacing $\underline{\boldsymbol{R}}^{\mathrm{BG}}$ by $\underline{\boldsymbol{M}}^{\mathrm{BG}} \otimes \underline{\boldsymbol{\nabla}}$ in 70 yields:

$$
\left(\underline{\boldsymbol{M}}^{\mathrm{BG}} \cdot \underline{\boldsymbol{\nabla}}\right) \cdot \underline{\boldsymbol{\nabla}}+p=0
$$

which means that $\boldsymbol{M}^{\mathrm{BG}}$ is actually in $\boldsymbol{S C}(p)$. It remains to show that ${\underset{\sim}{\boldsymbol{M}}}^{\mathrm{BG}}$ is the unique solution to the problem 47 . Consider any element $\underset{\sim}{\boldsymbol{M}} \in \boldsymbol{S C}(p)$, and set

$$
\boldsymbol{M}^{\prime}=\underset{\sim}{\boldsymbol{M}}-{\underline{\boldsymbol{M}^{\mathrm{BG}}}}^{\mathrm{B}}
$$

Clearly, $\boldsymbol{M}^{\prime}$ belongs to $S C(0)$ and, based on 63 , 64) and (68), we have by simple algebra:

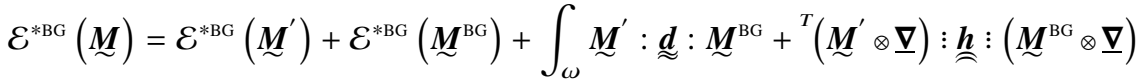

$$
\begin{aligned}
& =\mathcal{E}^{* \mathrm{BG}}\left(\underset{\sim}{\boldsymbol{M}^{\prime}}\right)+\mathcal{E}^{* \mathrm{BG}}\left({\underset{\sim}{\boldsymbol{B}}}^{\mathrm{BG}}\right)+\int_{\omega} \boldsymbol{M}^{\prime}:\left(\underline{\boldsymbol{\Phi}}^{\mathrm{BG}} \cdot \underline{\boldsymbol{\nabla}}\right)+{ }^{T}(\underset{\sim}{\boldsymbol{M}} \otimes \underline{\boldsymbol{\nabla}}):\left(\underline{\Phi}^{\mathrm{BG}}+\underset{\sim}{\boldsymbol{i}} \cdot \underline{\boldsymbol{\nabla}} W^{\mathrm{BG}}\right) .
\end{aligned}
$$

Recalling that the generalized displacements $\left(W^{\mathrm{BG}}, \boldsymbol{\Phi}^{\mathrm{BG}}\right)$ belong to $K C_{0}$ and that the stress field ${\underset{\sim}{\boldsymbol{M}}}^{\prime}$ belongs to $S C(0)$, integrating by parts implies that the last term of the right-hand side of (71) vanishes. Hence, we have:

$$
\mathcal{E}^{* \mathrm{BG}}(\underset{\sim}{\boldsymbol{M}})=\mathcal{E}^{* \mathrm{BG}}\left(\underline{\boldsymbol{M}}^{\prime}\right)+\mathcal{E}^{* \mathrm{BG}}\left(\underline{\boldsymbol{M}}^{\mathrm{BG}}\right)
$$

We end the proof by mentioning that $\mathcal{E}^{* \mathrm{Bg}}\left(\underset{\boldsymbol{M}^{\prime}}{)}\right)$ is positive as soon as $\boldsymbol{M}^{\prime}$ does not vanish.

Having studied the Bending-Gradient theory for clamped plates, we now introduce this theory when free boundary conditions are prescribed.

\section{Mathematical formulation of the Bending-Gradient problem for a loaded plate with free boundary conditions}

In this section, we study the problem with completely free boundary conditions. Since the shear force compliance tensor is assumed to be positive definite, these conditions write as follows when the generalized stress fields are regular enough (See [32], chapter 6):

$$
\underset{\sim}{\boldsymbol{M}}=0, \quad(\underset{\sim}{\boldsymbol{i}}: \underline{\boldsymbol{R}}) \cdot \underline{\boldsymbol{n}}=0, \text { on } \partial \omega,
$$

or in terms of components:

$$
M_{\alpha \beta}=0, \quad R_{\alpha \beta \beta} n_{\alpha}=0 \text {, on } \partial \omega .
$$

Here, $\underline{\boldsymbol{n}}$ is the outer normal vector to $\partial \omega$.

We hence have to find $W, \boldsymbol{\Phi}, \underset{\boldsymbol{\chi}}{\boldsymbol{T}} \boldsymbol{\Gamma}, \boldsymbol{M}$ and $\boldsymbol{R}$ solution of (28), 29, , 32, (33) and (72). This prompts us to introduce a displacement formulation (see 82 ) then to prove that the corresponding minimization problem admits a unique solution (see Theorem 4). We finally establish the relation between this formulation and a stress formulation (Theorem 6). 
5.1 Formulation of the problem

We introduce the set of the generalized Bending-Gradient displacements which are kinematically compatible with free boundary conditions as the quotient space:

$$
K C_{f}=K C / \mathcal{R},
$$

where

$$
K C=H^{1}(\omega) \times \underline{H}(\operatorname{div}, \omega),
$$

and $\mathcal{R}$ is the set of the displacements $(W, \Phi)$ in $K C$ such that the generalized BendingGradient strains $(\underset{\sim}{\boldsymbol{\chi}}, \underset{\sim}{\Gamma})$ are identically null.

Obviously, such displacements verify $\underline{\Phi}=-\underset{\sim}{\boldsymbol{i}} \cdot \underline{\boldsymbol{\nabla}} W$ and $\underline{\boldsymbol{\Phi}} \cdot \underline{\boldsymbol{\nabla}}=\left(-W_{, \alpha \beta}\right)=0$. Therefore, $W$ is an affine function of $x_{1}$ and $x_{2}$ and the set $\mathcal{R}$ is defined as follows:

$$
\mathcal{R}=\left\{(W, \underline{\mathbf{\Phi}}) \in K C / W=\underline{\boldsymbol{\alpha}} \cdot \underline{\boldsymbol{x}}+\beta, \underline{\boldsymbol{\Phi}}=\underset{\sim}{-\boldsymbol{i}} \cdot \underline{\boldsymbol{\alpha}}, \alpha_{1}, \alpha_{2}, \beta \in \mathbb{R}\right\} .
$$

In components, we have:

$$
W\left(x_{1}, x_{2}\right)=\alpha_{1} x_{1}+\alpha_{2} x_{2}+\beta,
$$

and

$$
\left(\Phi_{111}, \Phi_{221}, \Phi_{121}, \Phi_{112}, \Phi_{222}, \Phi_{122}\right)\left(x_{1}, x_{2}\right)=\left(-\alpha_{1}, 0,-\frac{1}{2} \alpha_{2}, 0,-\alpha_{2},-\frac{1}{2} \alpha_{1}\right) .
$$

Clearly, the space $\mathcal{R}$ is a closed subspace of $K C$. We can therefore consider the orthogonal projection of $K C$ on $\mathcal{R}$ according to the norm $\|\bullet\|_{K C}$ : For any $(W, \Phi) \in K C$, there exists a unique $\Pi(W, \Phi)$ such that:

$$
\|(W, \Phi)-\Pi(W, \Phi)\|_{K C}=\inf _{(V, \underline{\Psi}) \in \mathcal{R}}\|(W, \Phi)-(V, \Psi)\|_{K C} .
$$

Lemma 7 The quotient space $K_{f}$, endowed with the scalar product

$$
\left\langle[\breve{W}, \breve{\mathbf{\Phi}}],\left[\breve{W}^{\prime}, \breve{\mathbf{\Phi}}^{\prime}\right]\right\rangle_{K C_{f}}=\left\langle(W, \underline{\mathbf{\Phi}})-\Pi(W, \underline{\mathbf{\Phi}}),\left(W^{\prime}, \Phi^{\prime}\right)-\Pi\left(W^{\prime}, \Phi^{\prime}\right)\right\rangle_{K C},
$$

where $(W, \Phi)$ (resp. $\left(W^{\prime}, \Phi^{\prime}\right)$ ) is any element in $[\breve{W}, \breve{\Phi}]$ (resp. $\left[\breve{W}^{\prime}, \breve{\Phi}^{\prime}\right]$ ), is a Hilbert space.

Proof We start by showing that the scalar product 76 is well-defined. Consider $(W, \Phi)$ and $\left(W^{*}, \Phi^{*}\right)$ two elements in $(\breve{W}, \breve{\mathbf{\Phi}})$. Since $\Pi$ is a linear operator, we can write :

$\left(W^{*}, \Phi^{*}\right)-\Pi\left(W^{*}, \Phi^{*}\right)=\left(W^{*}, \underline{\Phi}^{*}\right)-(W, \underset{\Phi}{\Phi})-\Pi\left(\left(W^{*}, \Phi^{*}\right)-(W, \underline{\Phi})\right)+(W, \underline{\Phi})-\Pi(W, \Phi)$. Now, $\left(W^{*}, \Phi^{*}\right)-(W, \Phi)$ being an element of $\mathcal{R}$ yields :

$$
\left(W^{*}, \Phi^{*}\right)-(W, \Phi)-\Pi\left(\left(W^{*}, \Phi^{*}\right)-(W, \Phi)\right)=0 .
$$

We therefore have that:

$$
\left(W^{*}, \underline{\Phi}^{*}\right)-\Pi\left(W^{*}, \underline{\Phi}^{*}\right)=(W, \underline{\mathbf{\Phi}})-\Pi(W, \underline{\mathbf{\Phi}}),
$$


which means that the scalar product 76 is well-defined on $K C_{f}$.

We are now left to prove that $K C_{f}$ is a Hilbert space. To this end, let $\left[\breve{W}_{n}, \breve{\Phi}_{n}\right] \in K C_{f}$, $n \in \mathbb{N}$, be a Cauchy sequence in the norm $\|\bullet\|_{K C_{f}}$. Obviously, $\left(W_{n}, \Phi_{n}\right)-\Pi\left(W_{n}, \Phi_{n}\right)$ is a Cauchy sequence in $K C$. Hence, it converges to an element $\left(W_{\infty}, \Phi_{\infty}\right)$ such that :

$$
\Pi\left(W_{\infty}, \Phi_{\infty}\right)=0
$$

since the operator $\Pi$ is continuous. We now see that :

$$
\left\|\left[\breve{W}_{n}, \breve{\Phi}_{n}\right]-\left[\breve{W}_{\infty}, \breve{\boldsymbol{\Phi}}_{\infty}\right]\right\|_{K C_{f}}^{2}=\left\|\left(W_{n}, \Phi_{n}\right)-\left(W_{\infty}, \Phi_{\infty}\right)-\Pi\left(W_{n}, \Phi_{n}\right)\right\|_{K C}^{2},
$$

hence $\left[\breve{W}_{n}, \breve{\Phi}_{n}\right]$ converges to $\left[\breve{W}_{\infty}, \breve{\Phi}_{\infty}\right]$ in $K C_{f}$. This ends the proof.

In the absence of kinematic boundary conditions, the global equilibrium of the plate is impossible unless the applied transverse load $p$ is self-balanced. Namely,

$$
\forall(W, \Phi) \in \mathcal{R}, \quad b(W, \Phi)=\int_{\omega} p W=0 .
$$

The above equation is equivalent to the following standard conditions stipulating that the resultant applied force and moments are null:

$$
\int_{\omega} p=0, \quad \int_{\omega} p x_{1}=0, \quad \text { and } \quad \int_{\omega} p x_{2}=0 .
$$

We are now in position to introduce a variational formulation on $K C_{f}$. We introduce the following linear form:

$$
\forall[\breve{W}, \breve{\Phi}] \in K C_{f}, \quad \breve{b}([\breve{W}, \breve{\Phi}])=b(W, \underline{\Phi})=\int_{\omega} p W,
$$

where $(W, \Phi)$ is any element in $[\breve{W}, \breve{\Phi}]$. This makes sense because equations 77 ensure that the above right-hand side does not depend on the choice of $(W, \Phi)$. Assume that $p$ is in $L^{2}(\omega)$. Then, the linear form $b$ is continuous on $K C$, and we have for any $[\breve{W}, \breve{\Phi}] \in K C_{f}$ :

$$
\begin{aligned}
& |\breve{b}([\breve{W}, \breve{\mathbf{\Phi}}])|=|b((W, \boldsymbol{\Phi})-\Pi(W, \Phi))|
\end{aligned}
$$

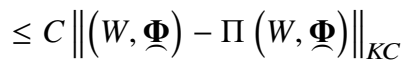

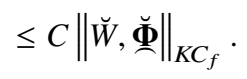

We conclude that the linear form $\breve{b}$ is continuous on $K C_{f}$. Let us now introduce the following bilinear form on $K_{f}$ :

$$
\begin{aligned}
& \breve{a}\left([\breve{W}, \underline{\mathbf{\Phi}}],\left[\breve{W}^{\prime}, \breve{\mathbf{\Phi}}^{\prime}\right]\right)=a\left((W, \underline{\boldsymbol{\Phi}}),\left(W^{\prime}, \underline{\Phi}^{\prime}\right)\right)=\int_{\omega}(\underline{\boldsymbol{\Phi}} \cdot \underline{\boldsymbol{\nabla}}): \underset{\approx}{\boldsymbol{D}}:\left(\underline{\boldsymbol{\Phi}}^{\prime} \cdot \underline{\boldsymbol{\nabla}}\right)
\end{aligned}
$$

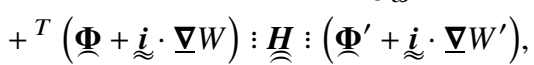


where $(W, \Phi)$ (resp. $\left.\left(W^{\prime}, \Phi^{\prime}\right)\right)$ is any element in $[\breve{W}, \breve{\Phi}]$ (resp. $\left.\left[\breve{W}^{\prime}, \breve{\Phi}^{\prime}\right]\right)$. The bilinear form $\breve{a}$ is well-defined on $K C_{f}$ because, obviously, all the elements of $[\breve{W}, \breve{\Phi}]$ have the same generalized strains $\Phi \cdot \underline{\nabla}$ and $\Phi+\underset{\sim}{\boldsymbol{i}} \cdot \underline{\boldsymbol{\nabla}} W$. Moreover, $\breve{a}$ is continuous on $K C_{f}$. Indeed,

$$
\begin{aligned}
\left|\breve{a}\left([\breve{W}, \breve{\Phi}],\left[\breve{W}^{\prime}, \breve{\Phi}^{\prime}\right]\right)\right| & =\left|a\left((W, \Phi)-\Pi(W, \Phi),\left(W^{\prime}, \Phi^{\prime}\right)-\Pi\left(W^{\prime}, \Phi^{\prime}\right)\right)\right| \\
& \leq C\|(W, \Phi)-\Pi(W, \Phi)\|_{K C}\left\|\left(W^{\prime}, \Phi^{\prime}\right)-\Pi\left(W^{\prime}, \Phi^{\prime}\right)\right\|_{K C} \\
& \leq C\|\breve{W}, \breve{\Phi}\|_{K C_{f}}\left\|\breve{W}^{\prime}, \breve{\Phi}^{\prime}\right\|_{K C_{f}} .
\end{aligned}
$$

We consider the following minimization problem:

$$
\min \left\{\breve{\mathscr{P}}([\breve{W}, \breve{\Phi}]), \quad[\breve{W}, \breve{\Phi}] \in K C_{f}\right\},
$$

where

$$
\breve{\mathcal{P}}([\breve{W}, \breve{\Phi}])=\frac{1}{2} \breve{a}([\breve{W}, \breve{\Phi}],[\breve{W}, \breve{\Phi}])-\breve{b}([\breve{W}, \breve{\Phi}]) \text {. }
$$

The well-posedness of this problem relies on the coercivity of the bilinear form $\breve{a}$, which we will prove in the following paragraph.

\subsection{Coercivity of the bilinear form $\breve{a}$}

In order to prove the coercivity of $\breve{a}$, we will need the following lemma:

Lemma 8 Let $[\breve{W}, \breve{\Phi}] \in K C_{f}$. There exist two constants $c>0$ and $c^{\prime}>0$ such that

$$
c\|\breve{W}, \breve{\Phi}\|_{N} \leq\|\breve{W}, \breve{\Phi}\|_{K C_{f}} \leq c^{\prime}\|\breve{W}, \breve{\Phi}\|_{N},
$$

where $\|\breve{W}, \breve{\Phi}\|_{N}^{2}=a_{N}((W, \Phi),(W, \Phi))$, with $a_{N}$ given by $[55$ and $(W, \Phi)$ is any element in $[\breve{W}, \breve{\Phi}]$, defines a norm on $K C_{f}$.

Proof We decompose the proof into three steps.

Step 1. Since all the elements of $[\breve{W}, \breve{\Phi}]$ have the same generalized strains $\Phi \cdot \underline{\nabla}$ and $\Phi+\underset{\sim}{\boldsymbol{i}} \cdot \underline{\boldsymbol{\nabla}} W$, one can define the bilinear form $\breve{a}_{N}$ on $K C_{f} \times K C_{f}$ as:

$$
\breve{a}_{N}\left([\breve{W}, \breve{\Phi}],\left[\breve{W}^{\prime}, \breve{\Phi}^{\prime}\right]\right)=a_{N}\left((W, \Phi),\left(W^{\prime}, \Phi^{\prime}\right)\right),
$$

where $(W, \Phi)$ (resp. $\left.\left(W^{\prime}, \Phi^{\prime}\right)\right)$ is any element in $[\breve{W}, \breve{\Phi}]$ (resp. $\left[\breve{W}^{\prime}, \breve{\Phi}^{\prime}\right]$ ). Since the bilinear form $\breve{a}_{N}$ is symmetric and positive, it only remains to prove that it is definite on $K C_{f}$ in order to show that it is a scalar product. Indeed, if we have $a_{N}((W, \Phi),(W, \Phi))=0$, then,

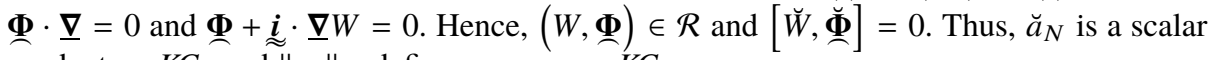
product on $K C_{f}$ and $\|, \cdot\|_{N}$ defines a norm on $K C_{f}$.

Step 2. We first start by proving that there exists a constant $c>0$ such that:

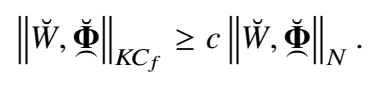


Let $(W, \Phi)$ be any element in $[\breve{W}, \breve{\Phi}]$. As in Section 4.3.1 we decompose the third-order tensor $\boldsymbol{\Phi}$ into its spherical part $\underline{\Phi}^{\mathrm{s}}=\underset{\sim}{\boldsymbol{i}} \cdot \underline{\boldsymbol{\Phi}}^{\mathrm{s}}$ and its deviatoric part $\underline{\Phi}^{\mathrm{d}}$. Hence, $\|W, \Phi\|_{N}$ can be written as follows:

$$
\begin{aligned}
\|\breve{W}, \underline{\mathbf{\Phi}}\|_{N}^{2} & =\|\underline{\boldsymbol{\Phi}} \cdot \underline{\boldsymbol{\nabla}}\|_{\underline{\boldsymbol{L}}^{2}(\omega)}^{2}+\|\underline{\boldsymbol{\Phi}}+\underset{\sim}{\boldsymbol{i}} \cdot \underline{\boldsymbol{\nabla}} W\|_{\underline{\mathbf{L}}^{2}(\omega)}^{2} \\
& =\|\underline{\boldsymbol{\Phi}} \cdot \underline{\boldsymbol{\nabla}}\|_{\underline{\mathbf{L}}^{2}(\omega)}^{2}+\left\|\underline{\boldsymbol{\Phi}}^{\mathrm{d}}\right\|_{\underline{\boldsymbol{L}}^{2}(\omega)}^{2}+\frac{3}{2}\left\|\underline{\boldsymbol{\Phi}}^{\mathrm{s}}+\underline{\boldsymbol{\nabla}} W\right\|_{\underline{\boldsymbol{L}}^{2}(\omega)}^{2}
\end{aligned}
$$

Besides, we have:

$$
\begin{aligned}
\|\breve{W}, \underline{\breve{\Phi}}\|_{K C_{f}}^{2} & =\left\|\left(W-W^{\mathcal{R}}, \underline{\mathbf{\Phi}}-\underline{\Phi}^{\mathcal{R}}\right)\right\|_{K C}^{2} \\
& =\left\|W-W^{\mathcal{R}}\right\|_{L^{2}(\omega)}^{2}+\left\|\underline{\nabla} W-\underline{\boldsymbol{\nabla}} W^{\mathcal{R}}\right\|_{\underline{\mathbf{L}}^{2}(\omega)}^{2} \\
& +\frac{3}{2}\left\|\underline{\Phi}^{\mathrm{s}}-\underline{\boldsymbol{\Phi}}^{\mathcal{R}}\right\|_{\underline{\boldsymbol{L}}^{2}(\omega)}^{2}+\left\|\underline{\boldsymbol{\Phi}}^{\mathrm{d}}\right\|_{\underline{\boldsymbol{L}}^{2}(\omega)}^{2}+\|\underline{\boldsymbol{\Phi}} \cdot \underline{\boldsymbol{\nabla}}\|_{\underline{\mathbf{L}}^{2}(\omega)}^{2},
\end{aligned}
$$

where we denote by $\left(W^{\mathcal{R}}, \Phi^{\mathcal{R}}\right)$ the orthogonal projection of $(W, \boldsymbol{\Phi})$ on $\mathcal{R}$, i.e.,

$$
\Pi(W, \underline{\Phi})=\left(W^{\mathcal{R}}, \underline{\Phi}^{\mathcal{R}}\right) \in \mathcal{R} .
$$

We can obviously write:

$$
\left\|\underline{\nabla} W-\underline{\boldsymbol{\nabla}} W^{\mathcal{R}}\right\|^{2}+\frac{3}{2}\left\|\underline{\boldsymbol{\Phi}}^{\mathrm{s}}-\underline{\boldsymbol{\Phi}}^{\mathcal{R}}\right\|^{2} \geq \frac{1}{2}\left\|\underline{\boldsymbol{\nabla}} W-\underline{\boldsymbol{\nabla}} W^{\mathcal{R}}\right\|^{2}+\frac{1}{2}\left\|\underline{\boldsymbol{\Phi}}^{\mathrm{s}}-\underline{\boldsymbol{\Phi}}^{\mathcal{R}}\right\|^{2} .
$$

By convexity of the square function, we obtain the following inequality:

$$
\frac{1}{2}\left\|\underline{\boldsymbol{\nabla}} W-\underline{\boldsymbol{\nabla}} W^{\mathcal{R}}\right\|^{2}+\frac{1}{2}\left\|\underline{\boldsymbol{\Phi}}^{\mathrm{s}}-\underline{\boldsymbol{\Phi}}^{\mathcal{R}}\right\|^{2} \geq\left\|\frac{1}{2}\left(\underline{\boldsymbol{\nabla}} W-\underline{\boldsymbol{\nabla}} W^{\mathcal{R}}+\underline{\boldsymbol{\Phi}}^{\mathrm{s}}-\underline{\boldsymbol{\Phi}}^{\mathcal{R}}\right)\right\|^{2} .
$$

Noticing that $\underline{\nabla} W^{\mathcal{R}}+\underline{\boldsymbol{\Phi}}^{\mathcal{R}}=0$ by the very definition of $\mathcal{R}$, then equation 85 and the above inequalities yield:

$$
\|\breve{W}, \underline{\mathbf{\Phi}}\|_{K C_{f}}^{2} \geq \frac{1}{4}\left\|\underline{\boldsymbol{\Phi}}^{\mathrm{s}}+\underline{\boldsymbol{\nabla}} W\right\|_{\underline{\mathbf{L}}^{2}(\omega)}^{2}+\left\|\underline{\Phi}^{\mathrm{d}}\right\|_{\underline{\mathbf{L}}^{2}(\omega)}^{2}+\|\underline{\boldsymbol{\Phi}} \cdot \underline{\boldsymbol{\nabla}}\|_{\underline{\mathbf{L}}^{2}(\omega)}^{2} \geq \frac{1}{6}\|\breve{W}, \underline{\mathbf{\Phi}}\|_{N}^{2} .
$$

Step 3. We are left with proving that there exists a constant $c^{\prime}>0$ such that:

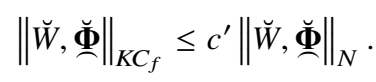

This is equivalent to the continuity of the injection of $K C_{f}$ equipped with the norm $\|\bullet\|_{N}$ into $K C_{f}$ equipped with the norm $\|\bullet\|_{K C_{f}}$. Considering a sequence $\left[\breve{W}_{k}, \breve{\Phi}_{k}\right] \in K C_{f}, k \in \mathbb{N}$ such that $\left\|\breve{W}_{k}, \breve{\Phi}_{k}\right\|_{N} \longrightarrow 0$ as $k$ goes to infinity, we have to prove that $\|\breve{W}, \breve{\Phi}\|_{K C_{f}} \longrightarrow 0$ as $k$ goes to infinity.

Using (84), we can see that :

$$
\left\|\underline{\boldsymbol{\Phi}}_{k}^{\mathrm{s}}+\underline{\boldsymbol{\nabla}} W_{k}\right\|_{\underline{\boldsymbol{L}}^{2}(\omega)}^{2} \longrightarrow 0
$$

and that :

$$
\left\|\underline{\Phi}_{k} \cdot \underline{\boldsymbol{\nabla}}\right\|_{\underline{L}^{2}(\omega)}^{2}+\left\|\underline{\boldsymbol{\Phi}}_{k}^{\mathrm{d}}\right\|_{\underline{L}^{2}(\omega)}^{2} \longrightarrow 0 .
$$


According to Lemma 4 and the above limit, for each $k$, there exists a vector field $\underline{\boldsymbol{r}}_{k}$ of the form (10) :

$$
\underline{\boldsymbol{r}}_{k}\left(x_{1}, x_{2}\right)=\left(u_{k}-w_{k} x_{2}, v_{k}+w_{k} x_{1}\right),
$$

such that:

$$
\left\|\underline{\Phi}_{k}^{\mathrm{s}}-\underline{\boldsymbol{r}}_{k}\right\|_{\underline{\boldsymbol{L}}^{2}(\omega)} \longrightarrow 0 .
$$

It follows from equations $(88)$ and $(90)$ that:

$$
\left\|\underline{\boldsymbol{r}}_{k}+\underline{\boldsymbol{\nabla}} W_{k}\right\|_{\underline{\underline{L}}^{2}(\omega)} \longrightarrow 0 .
$$

Then, according to Lemma 5. $w_{k} \longrightarrow 0$ and there exists a sequence of real numbers denoted by $z_{k}$ such that:

$$
\left\|W_{k}+u_{k} x_{1}+v_{k} x_{2}+z_{k}\right\|_{H^{1}(\omega)} \longrightarrow 0 .
$$

In turn, $w_{k} \longrightarrow 0$ and $(90)$ implies that :

$$
\left\|\underline{\Phi}_{k}^{\mathrm{s}}-\underline{\boldsymbol{r}}_{k}^{(u, v)}\right\|_{\underline{\boldsymbol{L}}^{2}(\omega)} \longrightarrow 0,
$$

where $\underline{\boldsymbol{r}}_{k}^{(u, v)}=\left(u_{k}, v_{k}\right)$. Using the definition of the norm $\|\bullet\|_{K C_{f}}$ and $\left(V_{k}, \underline{\Psi}_{k}\right)$ in $\mathcal{R}$ defined by:

we have:

$$
\left(V_{k}, \Psi_{k}\right)=\left(-u_{k} x_{1}-v_{k} x_{2}-z_{k}, \underset{\boldsymbol{i}}{\approx} \cdot \underline{\boldsymbol{r}}_{k}^{(u, v)}\right),
$$

$$
\left\|\breve{W}_{k}, \breve{\mathbf{\Phi}}_{k}\right\|_{K C_{f}} \leq\left\|\left(W_{k}, \underline{\Phi}_{k}\right)-\left(V_{k}, \underline{\Psi}_{k}\right)\right\|_{K C} \longrightarrow 0,
$$

thanks to equations $(92)$ and $(93)$ together with $(89)$. This ends the proof.

5.3 Well-posedness of the minimization problem for free boundary conditions

We have shown in the previous sections that the bilinear form $\breve{a}$ is continuous and coercive on the Hilbert space $K C_{f}$. Moreover, we have proved that the linear form $\breve{b}$ is continuous on $K C_{f}$. As all the assumptions of the Lax-Milgram theorem are verified, we have the following result:

Theorem 4 Assume that:

- the transverse load $p \in L^{2}(\omega)$ is such that (77) are satisfied;

- the symmetric stiffness tensors $\underset{\approx}{\boldsymbol{D}}$ and $\underset{\boldsymbol{H}}{\boldsymbol{H}}$ are positive definite.

Then, the minimization problem 82 has a unique solution denoted by $\left[\breve{W}^{\mathrm{BG}}, \breve{\mathbf{\Phi}}^{\mathrm{BG}}\right]$ which is also the unique solution of the following problem:

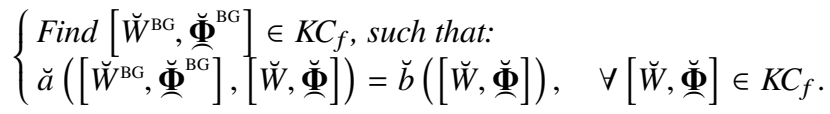

Our objective now is to build from $\left[\breve{W}^{\mathrm{BG}}, \breve{\Phi}^{\mathrm{BG}}\right]$ a solution to the problem formed by the equations (28), 29), (32), (33) and (72). To this end, we introduce the set $S_{0}(p)$ of statically compatible moment fields which are in equilibrium $(32)$ with the external load $p$ and with free boundary conditions (72), in the weak sense:

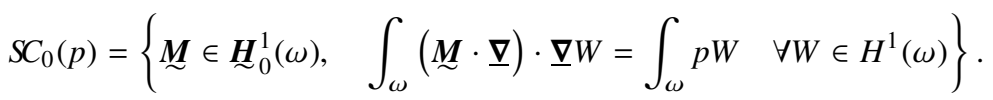

Indeed, using integration by parts, it easily seen that if $\underset{\sim}{\boldsymbol{M}} \in S_{0}(p)$ is regular enough, then the equations 32 and 72 will hold true in the strong sense with $\underset{\boldsymbol{R}}{\boldsymbol{R}} \boldsymbol{\boldsymbol { M }} \otimes \underline{\boldsymbol{\nabla}}$. 
Theorem 5 Suppose that the transverse load p and the stiffness tensors $\underset{\sim}{\boldsymbol{D}}$ and $\underset{\underline{\mathbf{H}} \text { satisfy the }}{ }$ assumptions of Theorem (4) and let $\left[\breve{W}^{\mathrm{BG}}, \breve{\Phi}^{\mathrm{BG}}\right]$ denote the unique solution to the minimization problem 82 . Let $\left(W^{\mathrm{BG}}, \Phi^{\mathrm{BG}}\right)$ be any element in $\left[\breve{W}^{\mathrm{BG}}, \breve{\mathbf{\Phi}}^{\mathrm{BG}}\right]$. The generalized strains associated to $\left(W^{\mathrm{BG}}, \Phi^{\mathrm{BG}}\right)$ through the compatibility conditions $(28,29$, are given by :

$$
\underline{\sim}^{\chi^{\mathrm{BG}}}=\underline{\boldsymbol{\Phi}}^{\mathrm{BG}} \cdot \underline{\boldsymbol{\nabla}}, \quad \text { and } \quad \underline{\Gamma}^{\mathrm{BG}}=\underline{\boldsymbol{\Phi}}^{\mathrm{BG}}+\underset{\sim}{\boldsymbol{i}} \cdot \underline{\boldsymbol{\nabla}} W^{\mathrm{BG}},
$$

and the generalized stress fields associated to $\left(W^{\mathrm{BG}}, \Phi^{\mathrm{BG}}\right)$ by the constitutive equations 33 are:

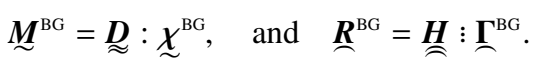

Then,

$$
{\underset{\sim}{\mathrm{BG}}}^{\mathrm{BG}} \in \mathrm{SC}_{0}(p), \quad \text { and } \quad \underline{\boldsymbol{R}}^{\mathrm{BG}}={\underset{\boldsymbol{M}}{\mathrm{BG}} \otimes}^{\mathrm{\nabla}} .
$$

Moreover, the above fields do not depend on the choice of $\left(W^{\mathrm{BG}}, \Phi^{\mathrm{BG}}\right)$ in $\left[\breve{W}^{\mathrm{BG}}, \breve{\boldsymbol{\Phi}}^{\mathrm{BG}}\right]$.

Proof The proof is similar to that of Theorem (3). Since $\left[\breve{W}^{\mathrm{BG}}, \breve{\mathbf{\Phi}}^{\mathrm{BG}}\right]$ satisfies 95 (we refer to Theorem (4)), we have that, for all $\left[\breve{W}^{\mathrm{BG}}, \breve{\boldsymbol{\Phi}}^{\mathrm{BG}}\right] \in K C_{f}$ :

$$
\int_{\omega} \underline{\boldsymbol{M}}^{\mathrm{BG}}:(\underline{\boldsymbol{\Phi}} \cdot \underline{\boldsymbol{\nabla}})+{ }^{T} \underline{\boldsymbol{R}}^{\mathrm{BG}}:(\underline{\boldsymbol{\Phi}}+\underset{\sim}{\boldsymbol{i}} \cdot \underline{\boldsymbol{\nabla}} W)=\int_{\omega} p W,
$$

where $\left(W^{\mathrm{BG}}, \underline{\boldsymbol{\Phi}}^{\mathrm{BG}}\right)$ is any element in $\left[\breve{W}^{\mathrm{BG}}, \breve{\boldsymbol{\Phi}}^{\mathrm{BG}}\right]$ and $(W, \underline{\boldsymbol{\Phi}})$ is any element of $K C$. By arguments similar to those used in the proof of Theorem 3 we obtain 99 .

\subsection{Equivalence to a stress formulation}

We have built in Theorem 5 the generalized stress fields $\left(\boldsymbol{M}^{\mathrm{BG}}, \boldsymbol{R}^{\mathrm{BG}}\right)$ solution to the problem with free boundary conditions. Moreover, we have proved that $\boldsymbol{M}^{\mathrm{BG}} \in \mathrm{SC}_{0}(p)$ and that $\underline{\boldsymbol{R}}^{\mathrm{BG}}=\underline{\boldsymbol{M}}^{\mathrm{BG}} \otimes \underline{\boldsymbol{\nabla}}$. We have the following result:

\section{Theorem 6}

Adopting the assumptions of Theorem 4 and the notations of Theorem 5 the stress field $\boldsymbol{M}^{\mathrm{BG}}$ defined in Theorem 5 is the unique solution to the problem:

$$
\min \left\{\mathcal{E}^{* \mathrm{BG}}=\int_{\omega} w^{* \mathrm{BG}}(\underset{\sim}{\boldsymbol{M}}, \underset{\sim}{\boldsymbol{M}} \otimes \underline{\boldsymbol{\nabla}}) d \omega, \quad \underset{\sim}{\boldsymbol{M}} \in \mathrm{SC}_{0}(p)\right\},
$$

where $w^{* \mathrm{BG}}$ is defined by (42).

Proof For a proof, we follow in close lines the second part of that of Theorem 3 where $S_{0}(p)$ is substituted to $S C(p)$. Consider any element $\underset{\sim}{\boldsymbol{M}} \in \mathrm{SC}_{0}(p)$, and set :

$$
\stackrel{\boldsymbol{M}}{ }^{\prime}=\underset{\sim}{\boldsymbol{M}}-{\underset{\sim}{\mathrm{MG}}}^{\mathrm{BG}} .
$$

Simple algebraic transformations lead to:

$$
\mathcal{E}^{* \mathrm{BG}}(\underset{\sim}{\boldsymbol{M}})=\mathcal{E}^{* \mathrm{BG}}\left({\underset{\boldsymbol{M}}{ }}^{\prime}\right)+\mathcal{E}^{* \mathrm{BG}}\left(\underline{\boldsymbol{M}}^{\mathrm{BG}}\right)+\int_{\omega} \boldsymbol{M}^{\prime}:\left(\underline{\boldsymbol{\Phi}}^{\mathrm{BG}} \cdot \underline{\boldsymbol{\nabla}}\right)+{ }^{T}\left(\underline{\boldsymbol{M}}^{\prime} \otimes \underline{\boldsymbol{\nabla}}\right):\left(\underline{\boldsymbol{\Phi}}^{\mathrm{BG}}+\underset{\sim}{\boldsymbol{i}} \cdot \underline{\boldsymbol{\nabla}} W^{\mathrm{BG}}\right) .
$$


Using that $\underset{\sim}{\boldsymbol{M}^{\prime}} \in S_{0}(0)$, integrating by parts leads to the fact that the last term in the above equation vanishes. We hence obtain that:

$$
\mathcal{E}^{* \mathrm{BG}}(\underset{\sim}{\boldsymbol{M}})=\mathcal{E}^{* \mathrm{BG}}\left({\underset{\boldsymbol{M}}{ }}^{\prime}\right)+\mathcal{E}^{* \mathrm{BG}}\left({\underset{\sim}{\mathrm{BG}}}^{\mathrm{BG}}\right)
$$

We end the proof by mentioning that $\mathcal{E}^{* \mathrm{Bg}}(\underset{\sim}{\boldsymbol{M}})$ is positive as soon as $\boldsymbol{M}^{\prime}$ does not vanish.

\section{Simple Support Boundary Conditions}

We have already presented the Bending-Gradient theory for clamped plates (section 4) as well as for free plates (section 5) and proved its well-posedness in both cases. Nevertheless, other types of boundary conditions can also be imposed on $\partial \omega$. In this section, we discuss the simple support boundary conditions.

According to [32], the soft simple support boundary conditions of the Bending-Gradient theory set to zero both the moment tensor $\underset{\sim}{\boldsymbol{M}}$ and the transverse displacement $W$. Namely:

$$
\underset{\sim}{\boldsymbol{M}}=0 \text {, and } W=0, \text { on } \partial \omega .
$$

Hence, these boundary conditions are a mixed between the clamped and the free boundary conditions. We can generalize the results established for the displacement formulation in Sect. 5 namely Theorems (1), 2) and (3) to the case of the mixed boundary conditions (102) as follows:

- The space $K C_{m}$ of kinematically compatible generalized displacement fields with mixed boundary conditions is defined as:

$$
K C_{m}=H_{0}^{1}(\omega) \times \underline{H}(\operatorname{div}, \omega) .
$$

Note that:

$$
K C_{0} \subset K C_{m} \subset K C .
$$

- The transverse load $p$ is assumed to be in $H^{-1}(\omega)$.

- The space $S C_{m}(p)$ of statically compatible generalized stress fields with mixed boundary conditions is defined as:

$$
S C_{m}(p)=\left\{\underset{\sim}{\boldsymbol{M}} \in{\underset{\sim}{\boldsymbol{H}}}_{0}^{1}(\omega), \quad M_{\alpha \beta, \alpha \beta}+p=0 \text { in the sense of distributions }\right\} .
$$

Note that:

$$
S_{0}(p) \subset S C_{m}(p) \subset S C(p)
$$

- The compliance tensors $\underset{\sim}{\boldsymbol{d}}$ and $\boldsymbol{h}$ are positive definite in the sense of (44) and (45).

- Theorem (1) holds true if $S C_{m}(\vec{p})$ and $S C_{m}(0)$ are respectively substituted for $S C(p)$ and $S C(0)$ in (47) and (48). The proof is exactly the same.

- Theorem (2) holds true if $K C_{m}$ is substituted for $K C_{0}$ in problem (52). The proof is mainly the same but we need to prove that there exists a constant $C^{\prime}$ such that:

$$
\forall(W, \Phi) \in K C_{m}, \quad\|W, \Phi\|_{K C_{m}} \leq C^{\prime}\|W, \Phi\|_{N} .
$$

To this aim, we proceed by contradiction as usual. Suppose that for all $k \in \mathbb{N}$, there exists $\left(W_{k}, \Phi_{k}\right) \in K C_{m}$ such that:

$$
\left\|W_{k}, \Phi_{k}\right\|_{K C_{m}}=1, \quad\left\|W_{k}, \Phi_{k}\right\|_{N} \leq \frac{1}{k}
$$


The expression of $\left\|W_{k}, \Phi_{k}\right\|_{N}$ is given by (59) and (60). From (104), we obtain (88) and (89). According to Lemma 4 and the limit (89), for each $k$, there exists a vector field $\underline{\boldsymbol{r}}_{k}$ of the form (10) such that 90 . Then, 91) follows from equations 88 and 90 and, according to Lemma 5 with $W_{k} \in H_{0}^{1}(\omega)$, we obtain the convergences $\underline{\boldsymbol{r}}_{k} \longrightarrow 0$ and $W_{k} \longrightarrow 0$ in $H_{0}^{1}(\omega)$. Hence, $\left(W_{k}, \Phi_{k}\right) \longrightarrow 0$ in $K C_{m}$ which is in contradiction with $\left\|W_{k}, \Phi_{k}\right\|_{K C_{m}}=1$.

- Theorem (3) which states the equivalence between the static and the kinematic approaches for clamped boundary conditions can be easily extended to prove the equivalence of these approaches for the mixed boundary conditions 102.

As mentioned earlier (see Sect. 3.2.4), the sixth order tensor $\boldsymbol{h}$ is positive but not definite on whole $\mathbb{R}$ in the general case. Sections 46 were devoted to the well-posedness of the Bending-Gradient problems when $\boldsymbol{h}$ is positive definite. It is time now to extend these results to the general case. Only clamped boundary conditions will be studied to avoid lengthening the paper.

\section{Formulation of the Bending-Gradient theory in the general case}

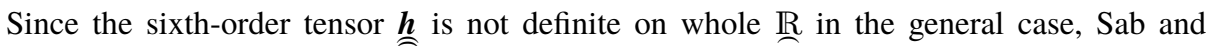

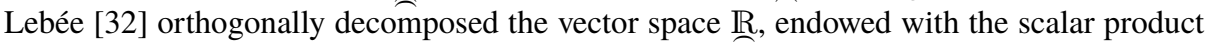
${ }^{T} \underline{X}: \underline{X}^{\prime}=X_{\alpha \beta \gamma} X_{\alpha \beta \gamma}^{\prime}$, into $\underline{\mathcal{S}}$ and its orthogonal $\underset{\mathcal{K}}{\mathcal{K}}$

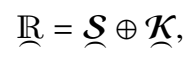

where $\mathcal{S}$ is the image of the sixth-order shear force compliance tensor $\underline{\boldsymbol{h}}$ :

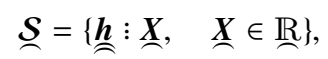

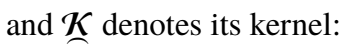

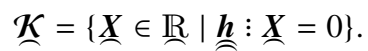

Let $\stackrel{\boldsymbol{P}}{\boldsymbol{S}}^{S}$ and $\stackrel{\boldsymbol{P}}{\boldsymbol{P}}^{K}$ denote, respectively, the orthogonal projection operator onto $\boldsymbol{\mathcal { S }}$ and $\mathcal{\mathcal { K }}$. It can be easily verified that any third-order tensor $\boldsymbol{X}$ can be written as:

$$
\underline{X}=\underline{X}^{S}+\underline{X}^{K}
$$

with

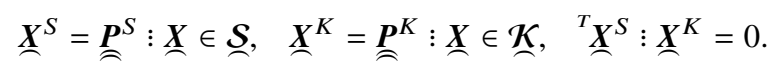

It is useful to notice that we always have:

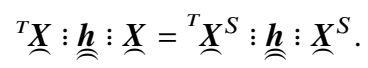

In other words, the shear force compliance tensor $\boldsymbol{h}$ is definite only on the subspace $\mathcal{S}$.

The subspace $\mathcal{S}$ contains all the spherical third-order tensors of the form (3) as shown by [32]. Hence, we have the following interesting property:

$$
\stackrel{\boldsymbol{P}^{S}}{\approx} \underset{\sim}{\boldsymbol{i}}=\underset{\approx}{\boldsymbol{i}}: \stackrel{\boldsymbol{P}}{ }^{S}=\underset{\sim}{\boldsymbol{i}}
$$

and the dimension of $\mathcal{S}$ is at least two. Actually, it is exactly two for homogeneous plates and in this case the Bending-Gradient theory degenerates into the Reissner-Mindlin theory. When $\underset{\boldsymbol{h}}{\boldsymbol{h}}$ is definite and therefore invertible, $\boldsymbol{\mathcal { S }}$ is equal to $\underset{\sim}{\mathbb{R}}$ of dimension six. In this case, 
$\underline{\boldsymbol{P}}^{\boldsymbol{S}}$ is the identity operator. So, the dimension of $\mathcal{S}$ is between two and six, depending on the elastic properties of the plate.

In order to take into account the possible non-definiteness of $\boldsymbol{h}$, both generalized rotation tensor $\boldsymbol{\Phi}$ and generalized shear force tensor $\boldsymbol{R}$ are enforced to belong to $\mathcal{S}$. The first equilibrium equation states that $\boldsymbol{R}$ is actually the projection of the bending gradient $\boldsymbol{M} \otimes \boldsymbol{\nabla}$ on $\mathcal{S}$. Moreover, using property (107), expressing the fact that $\mathcal{S}$ contains all the spherical third-order of the form (3), shows that $\underset{\sim}{\boldsymbol{i}}:(\underline{\boldsymbol{R}} \otimes \underline{\boldsymbol{\nabla}})=M_{\alpha \beta, \alpha \beta}$ in the general case, and hence the second equilibrium equation remains the same. Finally, since $\boldsymbol{h}$ may be not invertible, $\boldsymbol{H}$ will denote its Moore-Penrose pseudo inverse in the general case. In summary, the BendingGradient problem for clamped plates is to find $W$ and $\Phi \in \mathcal{S}$ solution of the following system of equations:

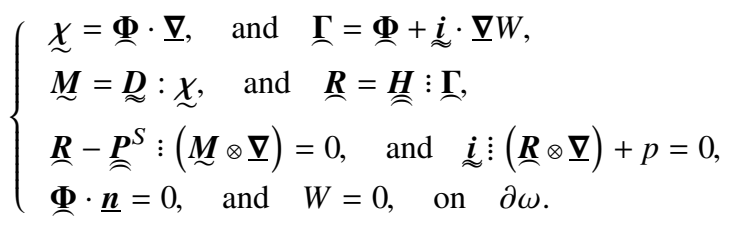

Note that since $\boldsymbol{\Phi}$ is in $\mathcal{S}$ and the spherical third-order tensors of the form 3 are also in $\mathcal{S}$, then $\boldsymbol{\Gamma}$ will be also in $\mathcal{S}$.

In general, the Bending-Gradient theory cannot be reduced to the Reissner-Mindlin theory. However, it was demonstrated that the Bending-Gradient theory coincides with the Reissner-Mindlin theory if, and only if, the shear force compliance tensor $\boldsymbol{h}$ has the following form:

$$
\stackrel{\boldsymbol{h}}{\stackrel{\boldsymbol{i}}{\boldsymbol{i}}} \cdot \underline{\boldsymbol{h}}^{\mathrm{RM}} \cdot \underset{\sim}{\boldsymbol{i}}
$$

where ${\underset{\boldsymbol{h}}{\boldsymbol{h} M}}^{\mathrm{RM}}$ denotes a positive definite second-order tensor called the Reissner-Mindlin shear force compliance tensor. This is exactly the case for homogeneous plates where $\underset{\boldsymbol{h}}{\boldsymbol{~}}$ is given by:

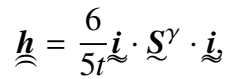

and ${\underset{\sim}{\boldsymbol{S}}}^{\gamma}=\left(4 S_{\alpha 3 \beta 3}\right)$ denotes the out-of-plane transverse shear compliance tensor.

Indeed, when $\boldsymbol{h}$ is of the above form, then $\boldsymbol{S}$ coincides with the set of spherical third-order tensors of the form (3). Hence, we have necessarily:

$$
\Phi=\underset{\sim}{\boldsymbol{i}} \cdot \underline{\varphi}
$$

where $\underline{\varphi}$ is a $2 \mathrm{D}$ vector representing rotations,

$$
\underline{\boldsymbol{R}}=\underline{\boldsymbol{P}}^{S}:(\underset{\sim}{\boldsymbol{M}} \otimes \underline{\boldsymbol{\nabla}})=\frac{2}{3} \underset{\sim}{\sim} \cdot(\underset{\sim}{\boldsymbol{M}} \cdot \underline{\boldsymbol{\nabla}}),
$$

and all the equations of the Reissner-Mindlin theory are retrieved.

We now define the proper functional spaces in which the stress formulation and the displacement formulation turn out to be well-posed in the general case where $\underset{\boldsymbol{h}}{=}$ may not be definite. 
7.1 Stress formulation

Let $(\underset{\sim}{\boldsymbol{M}}, \boldsymbol{R})$ be a generalized stress field defined over $\omega$. We recall that the complementary energy $\mathcal{E}^{* \mathrm{BG}}$ and the stress energy density $w^{* \mathrm{BG}}$ are respectively defined by (41) and (42). Assume that the shear force compliance $\stackrel{\boldsymbol{h}}{\Omega}$ is positive. Let $\mathcal{S}$ be its image and $\stackrel{\boldsymbol{P}}{S}^{\text {the }}$ orthogonal projection operator on $\mathcal{S}$. Assume that $(107)$ holds true. Then, the set of statically admissible generalized stress fields is defined as follows:

$$
S C^{\mathrm{BG}}(p)=\left\{(\underline{\boldsymbol{M}}, \underline{\boldsymbol{R}}) \in \underline{\sim}^{2} \times \underline{\boldsymbol{L}}^{2} / \quad \underline{\boldsymbol{R}}=\underline{\boldsymbol{P}}^{S}:(\underset{\sim}{\boldsymbol{M}} \otimes \underline{\boldsymbol{\nabla}}) \text { and } \underset{\sim}{\boldsymbol{i}}:(\underline{\boldsymbol{R}} \otimes \underline{\boldsymbol{\nabla}})+p=0\right\},
$$

where the equilibrium equations are in the sense of distributions, i.e., for all $(W, \Phi) \in$ $D(\omega) \times \boldsymbol{D}(\omega)$ :

$$
\int_{\omega} \underset{\boldsymbol{M}}{ }:\left(\left(\stackrel{\boldsymbol{P}}{ }^{S}: \underline{\boldsymbol{\Phi}}\right) \cdot \underline{\boldsymbol{\nabla}}\right)+{ }^{T} \underline{\boldsymbol{R}}:(\underline{\boldsymbol{\Phi}}+\underset{\sim}{\boldsymbol{i}} \cdot \underline{\boldsymbol{\nabla}} W)=\int_{\omega} p W .
$$

Note that the above definition of $S C^{\mathrm{BG}}(p)$ coincides with definition 46 of $S C(p)$ if $\underset{\boldsymbol{h}}{=}$ is definite and, hence, $\boldsymbol{P}^{S}$ is the identity operator.

The variational formulation of the Bending-Gradient problem consists in minimizing the

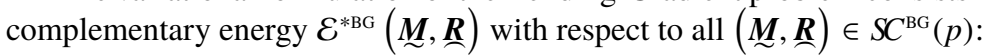

$$
\min \left\{\mathcal{E}^{* \mathrm{BG}}(\underset{\sim}{\boldsymbol{M}}, \underline{\boldsymbol{R}})=\int_{\omega} w^{* \mathrm{BG}}(\underset{\sim}{\boldsymbol{M}}, \underline{\boldsymbol{R}}), \quad(\underset{\sim}{\boldsymbol{M}}, \underline{\boldsymbol{R}}) \in \operatorname{SC}^{\mathrm{BG}}(p)\right\} .
$$

The following theorem states the well-posedness of the above minimization problem. Its proof is very similar to that of Theorem (1).

Theorem 7 Assume that: $p \in H^{-1}(\omega)$, the bending compliance tensor $\underset{\sim}{\mathbf{d}}$ is positive definite and the shear force compliance $\underline{\boldsymbol{h}}$ is positive. Let $\boldsymbol{S}$ be the image of $\underline{\boldsymbol{h}}$ and assume that 107) holds true where $\stackrel{\boldsymbol{P}}{\boldsymbol{S}}_{\text {is }}$ the orthogonal projection operator on $\underline{\mathcal{S}}$. Then, the minimization problem (111) admits a unique solution $\left({\underset{\boldsymbol{M}}{ }}^{\mathrm{BG}}, \underline{\boldsymbol{R}}^{\mathrm{BG}}\right) \in \operatorname{SC}^{\mathrm{BG}}(p)$. Moreover, $\left(\stackrel{\boldsymbol{M}}{\sim}^{\mathrm{BG}}, \underline{\boldsymbol{R}}^{\mathrm{BG}}\right)$ satisfies the following variational formulation:

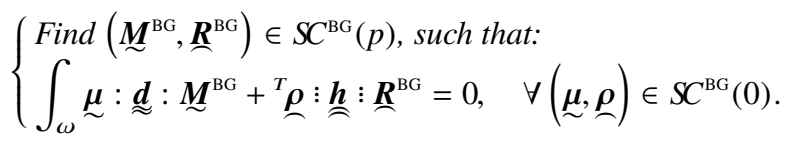

\subsection{Displacement formulation}

Let $(W, \boldsymbol{\Phi})$ be a generalized displacement field defined over $\omega$. We recall that the potential energy $\mathcal{E}^{\mathrm{BG}}$ and the strain energy density $w^{\mathrm{BG}}$ are respectively defined by (39) and (40). Because $\boldsymbol{h}$ is definite only on $\mathcal{S}$, the generalized rotation third-order tensor $\boldsymbol{\Phi}$ must lie in $\underline{H}_{0}(d i v, \omega)$ while satisfying $\boldsymbol{\Phi}\left(x_{1}, x_{2}\right) \in \mathcal{S}$ almost everywhere in $\omega$. In other words,

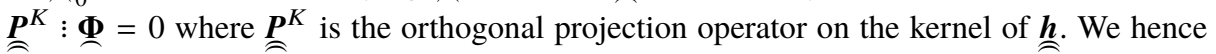
introduce the set $\widetilde{\Upsilon} \subseteq \underline{H}_{0}($ div,$\omega)$ defined by:

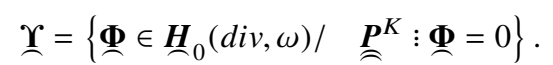

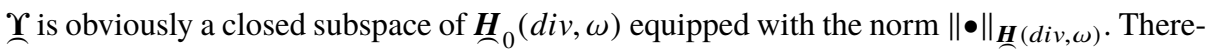
fore, $\Upsilon \mathfrak{\Upsilon}$ endowed with the scalar product $\langle\bullet\rangle_{\underline{H}(\operatorname{div}, \omega)}$ is a Hilbert space. 
As in section $4.3, W$ should belong to the Hilbert space $H_{0}^{1}(\omega)$. Always assuming that $p \in H^{-1}(\omega), \mathcal{E}^{\mathrm{BG}}$ introduced in 39 is well-defined for $(W, \Phi) \in H_{0}^{1}(\omega) \times \widetilde{\boldsymbol{\Phi}}$. We consider the minimization problem :

$$
\min \left\{\mathcal{E}^{\mathrm{BG}}(W, \underline{\Phi}), \quad(W, \underline{\Phi}) \in H_{0}^{1}(\omega) \times \underline{\Upsilon}\right\} .
$$

The following theorem states the well-posedness of the above minimization problem. Its proof is very similar to that of Theorems (2) and Theorem (3).

Theorem 8 Under the assumptions of Theorem (7), the minimization problem (113) is wellposed. Its unique solution is denoted by $\left(W^{\mathrm{BG}}, \Phi^{\mathrm{BG}}\right)$ and satisfies

$$
\left\|W^{\mathrm{BG}}, \underline{\Phi}^{\mathrm{BG}}\right\|_{H_{0}^{1}(\omega) \times \underline{H}(d i v, \omega)} \leq c\|p\|_{H^{-1}(\omega)},
$$

where $c$ is a strictly positive constant.

Moreover, the associated generalized stresses $(\underset{\sim}{\boldsymbol{M}}, \underset{\sim}{\boldsymbol{R}})$ obtained from $\left(W^{\mathrm{BG}}, \boldsymbol{\Phi}^{\mathrm{BG}}\right)$ via equations $108(a, b)$ are the unique solutions $\left(\boldsymbol{M}^{\mathrm{BG}}, \boldsymbol{R}^{\mathrm{BG}}\right)$ of the minimization problem (111).

We have seen in this section that when dealing with the Bending-Gradient problem in the general case, one has to introduce the kinematic constraint $\stackrel{P}{K}^{K} \boldsymbol{\Phi}=0$ when minimizing the potential energy. Another alternative is to regularize the shear force compliance tensor by adding to it a positive multiple of the identity operator. Indeed, the regularized tensor will be always definite and its inverse will be unambiguously defined. Hence, no need to introduce the kinematic constraint. The question that arises and that will be treated in the following section is the convergence of the regularized solution to the exact solution as the regularizing parameter goes to zero.

\section{Regularization of the shear force compliance tensor}

8.1 The regularized problem

Since the sixth-order tensor $\boldsymbol{h}$ is always positive, its definiteness is ensured by adding a positive multiple of the identity. The regularized tensor is denoted by $\underset{\curvearrowright}{\boldsymbol{h}}$ and defined by:

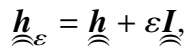

where $\varepsilon>0$. Clearly, $\underset{\sim}{\boldsymbol{h}}$ is always positive definite. Hence, in virtue of Section 4 , the regularized minimization problem for clamped boundary conditions :

$$
\min \left\{\mathcal{E}_{\varepsilon}^{*}(\underset{\sim}{\boldsymbol{M}}), \quad \boldsymbol{M} \in \operatorname{SC}(p)\right\},
$$

where

$$
\mathcal{E}_{\varepsilon}^{*}(\underset{\sim}{\boldsymbol{M}})=\frac{1}{2} \underset{\sim}{\boldsymbol{M}}: \underset{\sim}{\boldsymbol{d}}: \underset{\sim}{\boldsymbol{M}}+\frac{1}{2}^{T}(\underset{\sim}{\boldsymbol{M}} \otimes \underline{\boldsymbol{\nabla}}): \underset{\approx}{\boldsymbol{h}}:(\underset{\sim}{\boldsymbol{M}} \otimes \underline{\boldsymbol{\nabla}}),
$$

admits a unique solution ${\underset{\sim}{\boldsymbol{M}}}_{\varepsilon} \in S C(p)$. Furthermore, we denote $\underline{\boldsymbol{R}}_{\varepsilon}={\underset{\sim}{\boldsymbol{M}}}_{\boldsymbol{\varepsilon}} \otimes \underline{\boldsymbol{\nabla}}$. We know that there exists an unique $\left(W_{\varepsilon}, \Phi_{\varepsilon}\right) \in K C_{0}$ such that:

$$
{\underset{\sim}{\boldsymbol{M}}}_{\varepsilon}=\underset{\approx}{\boldsymbol{D}}: \underset{\sim}{\boldsymbol{\chi}}, \quad \underline{\boldsymbol{R}}_{\varepsilon}={\underset{\sim}{\boldsymbol{H}}}_{\varepsilon}: \underbrace{\boldsymbol{\Gamma}}_{\varepsilon}, \quad \underset{\sim}{\boldsymbol{\chi}}=\underbrace{\boldsymbol{\Phi}}_{\varepsilon} \cdot \underline{\boldsymbol{\nabla}}, \quad \underbrace{\boldsymbol{\Gamma}}_{\varepsilon}=\underbrace{}_{\varepsilon}+\underset{\approx}{\boldsymbol{i}} \cdot \underline{\nabla} W_{\varepsilon},
$$

where $\stackrel{\boldsymbol{H}}{\widetilde{H}}_{\varepsilon}$ is the inverse of $\underset{\widetilde{\boldsymbol{h}}}{\text {. }}$. 
8.2 Convergence of the regularized solution

The following theorem states the main result of this section:

Theorem 9 Under the assumptions of Theorem (7), there exists a unique solution of the

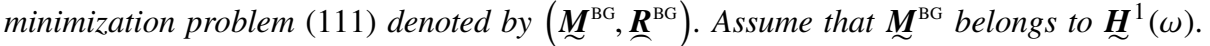
Then, ${\underset{\sim}{\boldsymbol{M}}}_{\varepsilon}$, the solution of the regularized problem $(115)$, converges in $\boldsymbol{L}^{2}(\omega)$ to $\boldsymbol{M}^{\mathrm{BG}}$, and $\underline{\boldsymbol{R}}_{\varepsilon}^{S}$ converges in $\underline{\boldsymbol{L}}^{2}(\omega)$ to $\underline{\boldsymbol{R}}^{\mathrm{BG}}$ when $\varepsilon$ tends to zero. Here, $\underline{\boldsymbol{R}}_{\varepsilon}=\boldsymbol{M}_{\varepsilon} \otimes \underline{\boldsymbol{\nabla}}$ and the notation (105) is used. Moreover, we have the convergence of the energies:

$$
\lim _{\varepsilon \rightarrow 0} \mathcal{E}_{\varepsilon}^{*}\left(\underline{\boldsymbol{M}}_{\varepsilon}\right)=\mathcal{E}^{* \mathrm{BG}}\left(\underline{\boldsymbol{M}}^{\mathrm{BG}}, \underline{\boldsymbol{R}}^{\mathrm{BG}}\right),
$$

and that:

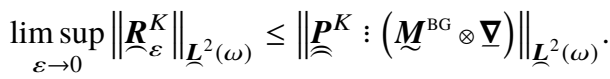

In addition, let $\left(W_{\varepsilon}, \Phi_{\varepsilon}\right) \in K C_{0}$ be associated to ${\underset{\sim}{\varepsilon}}_{\varepsilon}$ through 116. Then, $\left(W_{\varepsilon}, \Phi_{\varepsilon}\right)$ converges in $K_{0}$ to $\left(W^{\mathrm{BG}}, \Phi^{\mathrm{BG}}\right)$, the solution of the minimization problem (113), when $\varepsilon$ tends to zero.

Proof The hypothesis ${\underset{\sim}{\boldsymbol{M}}}^{\mathrm{BG}} \in{\underset{\sim}{\boldsymbol{H}}}^{1}(\omega)$ guarantees that $\boldsymbol{M}^{\mathrm{BG}}$ is in $\operatorname{SC}(p)$, and hence $\mathcal{E}_{\varepsilon}^{*}\left({\underset{\sim}{\boldsymbol{M}}}_{\varepsilon}\right) \leq$ $\mathcal{E}_{\varepsilon}^{*}\left({\underset{\boldsymbol{M}}{\mathrm{BG}}}^{\mathrm{BG}}\right)$ which can be written:

$$
\begin{aligned}
& \frac{1}{2} \int_{\omega}{\underset{\sim}{\boldsymbol{M}}}_{\varepsilon}: \underset{\sim}{\boldsymbol{d}}:{\underset{\sim}{\boldsymbol{M}}}_{\varepsilon}+{ }^{T} \underline{\boldsymbol{R}}_{\varepsilon}: \underset{\sim}{\boldsymbol{h}}: \underline{\boldsymbol{R}}_{\varepsilon}+\varepsilon^{T} \underline{\boldsymbol{R}}_{\varepsilon}: \underline{\boldsymbol{R}}_{\varepsilon} \leq
\end{aligned}
$$

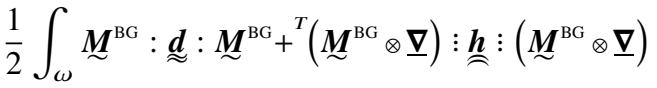

$$
\begin{aligned}
& +\varepsilon^{T}\left(\underline{\boldsymbol{M}}^{\mathrm{BG}} \otimes \underline{\boldsymbol{\nabla}}\right):\left(\underline{\boldsymbol{M}}^{\mathrm{BG}} \otimes \underline{\boldsymbol{\nabla}}\right) .
\end{aligned}
$$

By (106), we have that:

$$
{ }^{T} \underline{\boldsymbol{R}}_{\varepsilon}: \stackrel{\boldsymbol{h}}{\approx}: \underline{\boldsymbol{R}}_{\varepsilon}={ }^{T} \underline{\boldsymbol{R}}_{\varepsilon}^{S}: \underset{\overbrace{}}{\boldsymbol{h}}: \stackrel{\boldsymbol{R}}{\sim}_{\varepsilon}^{S},
$$

and

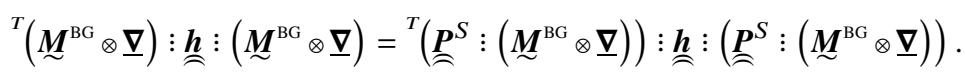

Recalling that:

$$
\stackrel{\boldsymbol{P}^{S}}{\lesssim}:\left(\underline{\boldsymbol{M}}^{\mathrm{BG}} \otimes \underline{\boldsymbol{\nabla}}\right)=\underline{\boldsymbol{R}}^{\mathrm{BG}},
$$

equation 119 can be written as:

$$
\begin{aligned}
& \frac{1}{2} \int_{\omega}{\underset{\sim}{\boldsymbol{M}}}_{\varepsilon}: \underset{\sim}{\boldsymbol{d}}:{\underset{\sim}{\boldsymbol{M}}}_{\varepsilon}+{ }^{T} \underline{\boldsymbol{R}}_{\varepsilon}^{S}: \underset{\sim}{\boldsymbol{h}}: \underline{\boldsymbol{R}}_{\varepsilon}^{S}+{ }_{\varepsilon}^{T} \underline{\boldsymbol{R}}_{\varepsilon}: \underline{\boldsymbol{R}}_{\varepsilon} \leq
\end{aligned}
$$

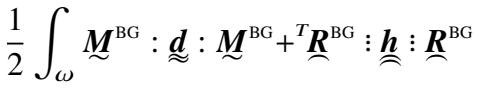

$$
\begin{aligned}
& +\varepsilon\left(\underline{\boldsymbol{M}}^{\mathrm{BG}} \otimes \underline{\boldsymbol{\nabla}}\right):\left(\underline{\boldsymbol{M}}^{\mathrm{BG}} \otimes \underline{\boldsymbol{\nabla}}\right) .
\end{aligned}
$$

Similarly, $\left(\underset{\varepsilon}{\boldsymbol{M}_{\varepsilon}}, \boldsymbol{R}_{\varepsilon}^{S}\right) \in S C^{\mathrm{BG}}(p)$ yields:

$$
\frac{1}{2} \int_{\omega} \boldsymbol{M}^{\mathrm{BG}}: \underset{\sim}{\boldsymbol{d}}:{\underset{\sim}{\mathrm{BG}}}^{\mathrm{BG}}+{ }^{T} \boldsymbol{R}^{\mathrm{BG}}: \underset{\sim}{\boldsymbol{h}}: \underline{\boldsymbol{R}}^{\mathrm{BG}} \leq \frac{1}{2} \int_{\omega}{\underset{\sim}{\boldsymbol{M}}}_{\varepsilon}: \underset{\sim}{\boldsymbol{d}}: \underset{\sim}{\boldsymbol{M}_{\varepsilon}}+{ }^{T} \underline{\boldsymbol{R}}_{\varepsilon}^{S}: \underset{\sim}{\boldsymbol{h}}: \underline{\boldsymbol{R}}_{\varepsilon}^{S} .
$$


Comparing inequalities (120) and 121) leads to the following inequality:

$$
\frac{1}{2} \int_{\omega} \varepsilon^{T} \underline{\boldsymbol{R}}_{\varepsilon}: \underline{\boldsymbol{R}}_{\varepsilon} \leq \frac{1}{2} \int_{\omega} \varepsilon{ }^{T}\left(\stackrel{\boldsymbol{M}}{ }^{\mathrm{BG}} \otimes \underline{\boldsymbol{\nabla}}\right):\left(\stackrel{\boldsymbol{M}}{ }^{\mathrm{BG}} \otimes \underline{\boldsymbol{\nabla}}\right) .
$$

Simplifying by $\varepsilon$, we obtain that:

$$
\left\|\underline{\boldsymbol{R}}_{\boldsymbol{\varepsilon}}\right\|_{\underline{\boldsymbol{L}}^{2}(\omega)} \leq\left\|\underline{\boldsymbol{M}}^{\mathrm{BG}} \otimes \underline{\boldsymbol{\nabla}}\right\|_{\underline{\boldsymbol{L}}^{2}(\omega)}<C .
$$

The above equation implies that:

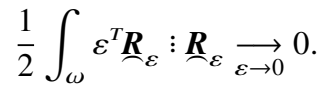

Then, taking the lim sup of inequality (120) and the lim inf of inequality (121), as $\epsilon$ goes to zero, results in the convergence of the energies (117) and in:

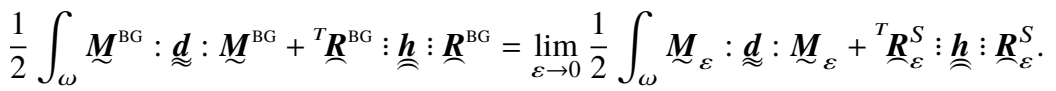

Because $\left({\underset{\sim}{\boldsymbol{M}}}^{\mathrm{BG}}, \underline{\boldsymbol{R}}^{\mathrm{BG}}\right)$ achieves the minimum of the complementary energy (111), we conclude that $\boldsymbol{M}_{\varepsilon}$ converges to ${\underset{\boldsymbol{M}}{\mathrm{BG}}}^{\mathrm{Bg}}$ in $\underline{\sim}^{2}(\omega)$ and that $\underline{\boldsymbol{R}}_{\varepsilon}^{\boldsymbol{S}}$ converges to $\underline{\boldsymbol{R}}^{\mathrm{BG}}$ in $\underline{\boldsymbol{L}}^{2}(\omega)$.

Finally, using the orthogonal decomposition (105), equation (123) can be written as:

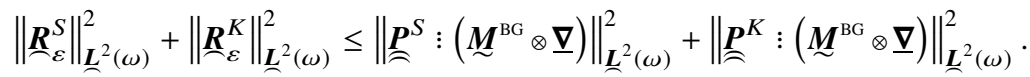

As

$$
\lim _{\varepsilon \rightarrow 0}\left\|\underline{\boldsymbol{R}}_{\varepsilon}^{S}\right\|_{\underline{\underline{L}}^{2}(\omega)}=\left\|\underline{\boldsymbol{R}}^{\mathrm{BG}}\right\|_{\underline{\boldsymbol{L}}^{2}(\omega)}=\left\|\underline{\boldsymbol{P}}^{S}:\left({\underset{\boldsymbol{M}}{\mathrm{BG}} \otimes}_{\boldsymbol{\nabla}}\right)\right\|_{\underline{\boldsymbol{L}}^{2}(\omega)},
$$

then, we obtain (118).

Now, according to Lemma 6 , the convergence of $\left(W_{\varepsilon}, \Phi_{\varepsilon}\right)$ to $\left(W^{\mathrm{BG}}, \Phi^{\mathrm{BG}}\right)$ in $K C_{0}$ is equivalent to:

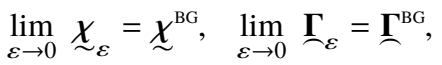

where the limits are, respectively, in $\underline{\boldsymbol{L}}^{2}(\omega)$ and $\underline{\boldsymbol{L}}^{2}(\omega)$, and ${\underset{\sim}{\boldsymbol{\chi}}}^{\mathrm{BG}}$ and $\underline{\boldsymbol{\Gamma}}^{\mathrm{BG}}$ are given by:

$$
\underline{\sim}^{\chi^{\mathrm{BG}}}=\underline{\boldsymbol{\Phi}}^{\mathrm{BG}} \cdot \underline{\boldsymbol{\nabla}}, \quad \underline{\boldsymbol{\Gamma}}^{\mathrm{BG}}=\underline{\boldsymbol{\Phi}}^{\mathrm{BG}}+\underset{\boldsymbol{i}}{\boldsymbol{i}} \cdot \underline{\boldsymbol{\nabla}} W^{\mathrm{BG}} .
$$

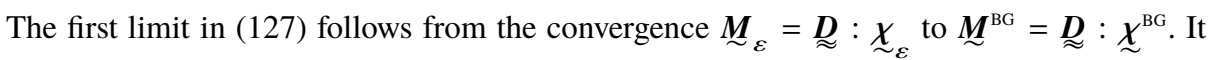
remains to prove the second limit. We will use the following properties of $\underline{\boldsymbol{H}}_{\varepsilon}$ which can be easily established by performing the singular value decomposition of $\underset{\widetilde{h}}{\boldsymbol{h}}$ and $\underset{\widetilde{\boldsymbol{H}}}{\boldsymbol{E}}$ :

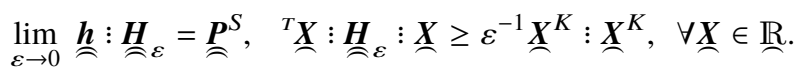

From the convergence of $\underline{\boldsymbol{R}}_{\varepsilon}^{S}$ to $\underline{\boldsymbol{R}}^{\mathrm{BG}}$, we have:

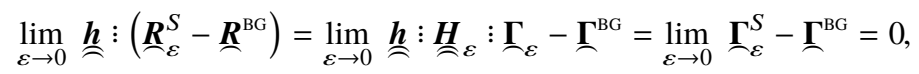

where the limits are in $\underline{\boldsymbol{L}}^{2}(\omega)$-norm. To end the proof, we notice that:

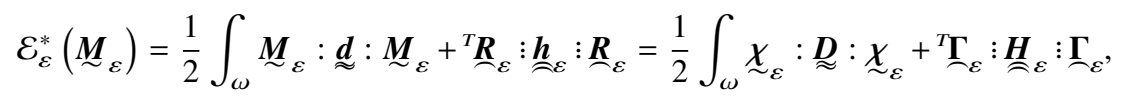

and hence,

$$
\mathcal{E}_{\varepsilon}^{*}\left({\underset{\sim}{\varepsilon}}_{\varepsilon}\right) \geq \frac{1}{2} \int_{\omega} \varepsilon^{-1^{T}} \underline{\Gamma}_{\varepsilon}^{K}: \Gamma_{\varepsilon}^{K}
$$

Then, using convergence (117) and the above inequality, we obtain the convergence to zero of $\boldsymbol{\Gamma}_{\varepsilon}^{K}$ in $\underline{\boldsymbol{L}}^{2}(\omega)$, and finally the second convergence in 127 . 


\section{Conclusion}

In this paper, a detailed mathematical justification for the recent theory of the BendingGradient elasticity was provided. Using variational methods, a series of existence and uniqueness theorems were formulated and proved. Since the shear force compliance tensor is not always definite, our study was divided into two parts. In the first part, we considered the particular case where the sixth-order tensor is positive definite. In this context, we defined the functional spaces to which the variables of the Bending-Gradient theory belong. Thereafter, using results established by [33], we rigorously proved that the stress and displacement minimization problems are well-posed when the plate under consideration is clamped or free. A brief discussion was then conducted concerning the simple support boundary conditions.

The second part of the paper was devoted to the extension of our findings to the general case. Some additional considerations had to be made when choosing the functional spaces. We investigated only the case of a clamped plate. The main conclusion is the existence and uniqueness of the solution to the Bending-Gradient problem.

An important point is that, in general, the Bending-Gradient theory cannot be reduced to a Reissner-Mindlin theory. However, when the plate is homogeneous, the Bending-Gradient theory corresponds to a Reissner-Mindlin theory. This case falls in the situations where the generalized shear force stiffness is not definite and was covered in Section 7

It is worthwhile to point out that adding a positive multiple of the identity operator to the shear force compliance tensor renders it definite. Indeed, in this case, the regularized problem admits a unique solution. Our main concern was to prove that the regularized solution converges to the exact solution of the Bending-Gradient in the general case. We managed to establish this fundamental result by assuming that the gradient of the exact solution to the Bending-Gradient stress problem is a square integrable function.

\section{Appendix}

In the sequel we use the symbol "c" to denote a generic positive constant. In other words, the constant $\mathrm{c}$ takes different values at successive occurrences, even in the same equation.

A.1

We denote by $\|\bullet\|_{S C(0)}$ the norm associated with the scalar product $\langle\bullet, \bullet\rangle_{S C(0)}$. Let ${\underset{\sim}{\boldsymbol{\mu}}}_{n} \in S C(0), n \in \mathbb{N}$ be a Cauchy sequence in the norm $\|\bullet\|_{S C(0)}$. Then, $\left({\underset{\sim}{\boldsymbol{\mu}}}_{n}\right)$ is a Cauchy sequence in $\underline{\boldsymbol{L}}^{2}(\omega)$ and $\left({\underset{\sim}{\boldsymbol{\mu}}}_{n}^{\otimes} \underline{\boldsymbol{\nabla}}\right)$ is a

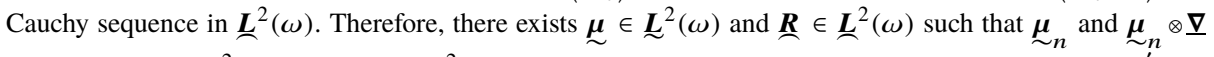
converge to $\underset{\sim}{\boldsymbol{\mu}}$ in ${\underset{\boldsymbol{L}}{\boldsymbol{L}}}^{2}(\omega)$ and to $\underline{\boldsymbol{R}}$ in $\underline{\boldsymbol{L}}^{2}(\omega)$, respectively. We thus have that ${\underset{\sim}{\boldsymbol{\mu}}}_{n}$ converges to $\underset{\sim}{\boldsymbol{\mu}}$ in ${\underset{\boldsymbol{D}}{ }}^{\prime}(\omega)$. Consequently, ${\underset{\sim}{\boldsymbol{\mu}}}_{n} \otimes \underline{\boldsymbol{\nabla}}$ converges to ${\underset{\sim}{\boldsymbol{\mu}} \otimes}_{\otimes} \boldsymbol{\nabla}$ in $\underline{\boldsymbol{D}}^{\prime}(\omega)$. Since ${\underset{\sim}{\boldsymbol{\mu}}}_{n} \otimes \underline{\boldsymbol{\nabla}}$ also converges to $\underline{\boldsymbol{R}}$ in $\underline{\boldsymbol{D}}^{\prime}(\omega)$, then we have

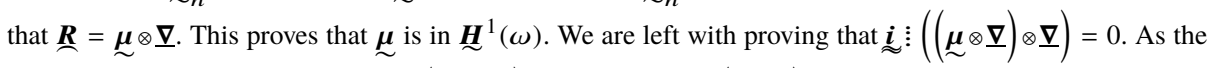
gradient operator is continuous, then $\left({\underset{\boldsymbol{\mu}}{n}}_{n} \otimes \underline{\boldsymbol{\nabla}}\right) \otimes \underline{\boldsymbol{\nabla}}$ converges to $(\underline{\boldsymbol{\mu}} \otimes \underline{\boldsymbol{\nabla}}) \otimes \underline{\boldsymbol{\nabla}}$ in the sense of distributions. We also have that ${\underset{\sim}{\boldsymbol{\mu}}}_{n} \in S C(0)$. Therefore, $\underset{\sim}{\boldsymbol{i}} \vdots\left(\left(\underline{\boldsymbol{\mu}}^{\otimes} \underline{\boldsymbol{\nabla}}\right) \otimes \underline{\boldsymbol{\nabla}}\right)=0$. We have proved that $S C(0)$ equipped with the scalar product $\langle\bullet, \bullet\rangle_{S C(0)}$ is hence a Hilbert space. 
A. 2

Let $\left(\underset{\sim}{\boldsymbol{M}},{\underset{\boldsymbol{M}}{ }}^{\prime}\right)$ be in ${\underset{\boldsymbol{H}}{ }}^{1}(\omega) \times{\underset{\boldsymbol{H}}{ }}^{1}(\omega)$. Then, using properties 44 ) and 45 and the Cauchy-Schwarz inequality, we have that:

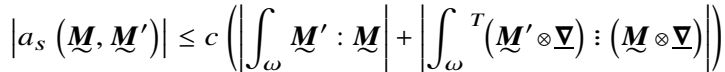

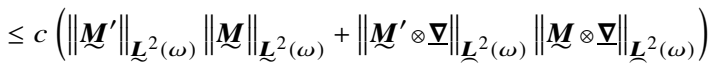

$$
\begin{aligned}
& \leq c\left(\left\|\boldsymbol{M}_{\sim}^{\prime}\right\|_{\underline{\boldsymbol{L}}^{2}(\omega)}^{2}+\left\|{\underset{\boldsymbol{M}}{\sim}}^{\prime} \otimes \underline{\boldsymbol{\nabla}}\right\|_{\underline{\boldsymbol{L}}^{2}(\omega)}^{2}\right)^{1 / 2}\left(\|\underset{\boldsymbol{M}}{\sim}\|_{\underline{\mathbf{L}}^{2}(\omega)}^{2}+\|\boldsymbol{M} \otimes \underline{\boldsymbol{\nabla}}\|_{\underline{\mathbf{L}}^{2}(\omega)}^{2}\right)^{1 / 2} \\
& \leq c\left\|\boldsymbol{M}^{\prime}\right\|_{\underline{\underline{H}}^{1}(\omega)}\|\boldsymbol{M}\|_{\underline{\boldsymbol{H}}^{1}(\omega)}
\end{aligned}
$$

We thus have proved that the bilinear form $a_{S}$ is continuous on $S C(0)$ and the linear map $\underset{\boldsymbol{\mu}}{\longrightarrow} a_{S}(\underset{\boldsymbol{P}}{\boldsymbol{\mu}})$ is also continuous on $S C(0)$.

A. 3

We have:

$$
\begin{aligned}
|b(W, \Phi)| & =\left|\int_{\omega} p W\right| \\
& \leq\|p\|_{H^{-1}(\omega)}\|W\|_{H_{0}^{1}(\omega)} \\
& \leq\|p\|_{H^{-1}(\omega)}\|W, \Phi\|_{K C_{0}} .
\end{aligned}
$$

Consequently, the linear form $b$ is continuous on $K C_{0}$.

A. 4

Using properties 44, and 45 then the Cauchy-Schwarz inequality leads to :

$$
\begin{aligned}
& \left|a\left((W, \underline{\boldsymbol{\Phi}}),\left(W^{\prime}, \underline{\boldsymbol{\Phi}}^{\prime}\right)\right)\right| \leq\left|\int_{\omega}(\underline{\boldsymbol{\Phi}} \cdot \underline{\boldsymbol{\nabla}}): \underset{\approx}{\boldsymbol{D}}:\left(\underline{\boldsymbol{\Phi}}^{\prime} \cdot \underline{\boldsymbol{\nabla}}\right)\right|+\left|\int_{\omega} \boldsymbol{T}(\underline{\boldsymbol{\Phi}}+\underset{\sim}{\boldsymbol{i}} \cdot \underline{\boldsymbol{\nabla}} W): \underset{\widetilde{\boldsymbol{H}}}{\approx}:\left(\underline{\boldsymbol{\Phi}}^{\prime}+\underset{\sim}{\boldsymbol{i}} \cdot \underline{\boldsymbol{\nabla}} W^{\prime}\right)\right| \\
& \leq c\left(\left|\int_{\omega}(\underline{\boldsymbol{\Phi}} \cdot \underline{\boldsymbol{\nabla}}):\left(\underline{\Phi}^{\prime} \cdot \underline{\boldsymbol{\nabla}}\right)\right|+\left|\int_{\omega} T(\underline{\boldsymbol{\Phi}}+\underset{\sim}{\boldsymbol{i}} \cdot \underline{\boldsymbol{\nabla}} W):\left({\underline{\Phi^{\prime}}}^{\boldsymbol{i}} \cdot \underline{\boldsymbol{\nabla}} W^{\prime}\right)\right|\right)
\end{aligned}
$$

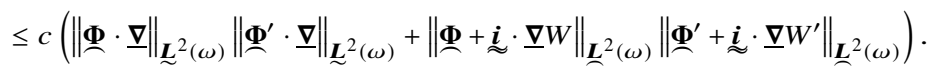

Since $\|\underline{\boldsymbol{i}} \cdot \underline{\nabla} W\|_{\underline{\underline{L}}^{2}(\omega)}^{2}=\frac{3}{2}\|\underline{\nabla} W\|_{\underline{\underline{L}}^{2}(\omega)}^{2}$, using the Poincaré inequality yields:

$$
\left|a\left((W, \Phi),\left(W^{\prime}, \Phi^{\prime}\right)\right)\right| \leq c\|W, \Phi\|_{K C_{0}}\left\|W^{\prime}, \Phi^{\prime}\right\|_{K C_{0}} .
$$

This ends the proof.

\section{References}

1. Alessandrini, S.M., Arnold, D.N., Falk, R.S., Madureira, A.L.: Derivation and Justification of Plate Models by Variational Methods. In: M. Fortin (ed.) Plates and shells, vol. 00, pp. 1-21. American Mathematical Society, Providence, RI, United States (1999)

2. Arnold, D.N., Falk, R.S.: Asymptotic Analysis of the Boundary Layer for the Reissner-Mindlin Plate Model. SIAM Journal on Mathematical Analysis 27(2), 486-514 (1996) 
3. Arnold, D.N., Madureira, A.L., Zhang, S.: On the Range of Applicability of the Reissner-Mindlin and Kirchhoff-Love Plate Bending Models. Journal of Elasticity 67(3), 171-185 (2002)

4. Bollé, L.: Contribution au problème linéaire de flexion d'une plaque élastique. Bulletin technique de la Suisse romande 73(21), 281-285 (1947)

5. Braess, D., Sauter, S., Schwab, C.: On the Justification of Plate Models. Journal of Elasticity 103(1), 53-71 (2010)

6. Carrera, E.: Theories and finite elements for multilayered, anisotropic, composite plates and shells. Archives of Computational Methods in Engineering 9(2), 87-140 (2002)

7. Ciarlet, P.G.: Plates and Junctions in Elastic Multi-structures: An Asymptotic Analysis. Collection Recherches en math\{é\}matiques appliqu\{é\}es. Masson (1990)

8. Ciarlet, P.G.: Mathematical Elasticity - Volume II: Theory of Plates. Elsevier Science Bv (1997)

9. Ciarlet, P.G., Destuynder, P.: Justification Of The 2-Dimensional Linear Plate Model. Journal de Mecanique 18(2), 315-344 (1979)

10. Diaz Diaz, A.: Un modèle de stratifiés. Comptes Rendus de l'Académie des Sciences - Series IIB Mechanics 329(12), 873-879 (2001)

11. Hencky, H.: Über die Berücksichtigung der Schubverzerrung in ebenen Platten. Ingenieur-Archiv 16(1), 72-76 (1947)

12. Hughes, T.J.R.: The Finite Element Method: Linear Static and Dynamic Finite Element Analysis. Dover Civil and Mechanical Engineering. Dover Publications (1987)

13. Lebée, A., Sab, K.: A Bending-Gradient model for thick plates. Part I: Theory. International Journal of Solids and Structures 48(20), 2878-2888 (2011)

14. Lebée, A., Sab, K.: A Bending-Gradient model for thick plates, Part II: Closed-form solutions for cylindrical bending of laminates. International Journal of Solids and Structures 48(20), 2889-2901 (2011)

15. Lebée, A., Sab, K.: Homogenization of cellular sandwich panels. Comptes Rendus Mécanique 340(4-5), 320-337 (2012)

16. Lebée, A., Sab, K.: Homogenization of thick periodic plates: Application of the Bending-Gradient plate theory to a folded core sandwich panel. International Journal of Solids and Structures 49(19-20), 27782792 (2012)

17. Lebée, A., Sab, K.: Homogenization of a space frame as a thick plate: Application of the Bending-Gradient theory to a beam lattice. Computers \& Structures 127, 88-101 (2013)

18. Lebée, A., Sab, K.: Justification of the Bending-Gradient Theory Through Asymptotic Expansions. In: H. Altenbach, S. Forest, A. Krivtsov (eds.) Generalized Continua as Models for Materials, pp. 217-236. Springer-Verlag Berlin Heidelberg (2013)

19. Lebée, A., Sab, K.: On the Generalization of Reissner Plate Theory to Laminated Plates, Part I: Theory. Journal of Elasticity 126(1), 39-66 (2017)

20. Lebée, A., Sab, K.: On the Generalization of Reissner Plate Theory to Laminated Plates, Part II: Comparison with the Bending-Gradient Theory. Journal of Elasticity 126(1), 67-94 (2017)

21. Morgenstern, D.: Herleitung der Plattentbeorie aus der dreidimensionalen Elastizitätstheorie. Archive for Rational Mechanics and Analysis 4, 145-152 (1959)

22. Neff, P., Hong, K.I., Jeong, J.: The Reissner-Mindlin plate is the $\Gamma$-limit of Cosserat elasticity. Mathematical Models and Methods in Applied Sciences 20(09), 1553-1590 (2010)

23. Noor, A.K., Malik, M.: An assessment of five modeling approaches for thermo-mechanical stress analysis of laminated composite panels. Computational Mechanics 25(1), 43-58 (2000)

24. Pagano, N.J.: Exact Solutions for Composite Laminates in Cylindrical Bending. Journal of Composite Materials 3(3), 398-411 (1969)

25. Pagano, N.J.: Influence of Shear Coupling in Cylindrical. Bending of Anisotropic Laminates. Journal of Composite Materials 4(3), 330-343 (1970)

26. Paroni, R., Podio-guidugli, P., Tomasseti, G.: A justification of Reissner-Mindlin plate theory through variational convergence. Analysis and Applications 05(02), 165-182 (2007)

27. Prager, W., Synge, J.: Approximations in elasticity based on the concept of function space. Quarterly of Applied Mathematics 5, 241-269 (1947)

28. Reddy, J.N.: On refined computational models of composite laminates. International Journal for Numerical Methods in Engineering 27(2), 361-382 (1989)

29. Reissner, E.: On the theory of bending of elastic plates. J. Math. Phys. 23, 184-191 (1944)

30. Reissner, E.: The effect of transverse shear deformation on the bending of elastic plates. Journal of Applied Mechanics 12(2), 69-77 (1945)

31. Reissner, E.: Reflections on the theory of elastic plates. Applied Mechanics Reviews 38(11), 1453 (1985)

32. Sab, K., Lebée, A.: Homogenization of Thick and Heterogeneous Plates. Wiley-ISTE (2015) 
33. Sab, K., Legoll, F., Forest, S.: Stress Gradient Elasticity Theory: Existence and Uniqueness of Solution. Journal of Elasticity 123(2), 179-201 (2016)

34. Whitney, J.M.: Stress Analysis of Thick Laminated Composite and Sandwich Plates. Journal of Composite Materials 6(4), 426-440 (1972) 